\title{
Spaces of Curvature Bounded Above
}

S. Buyalo* and V. Schroeder

$\begin{array}{ll}\text { 1. Motivation } & 296\end{array}$

2. Defining CBA 297

2.1. CBA and CAT spaces $\quad 297$

2.2. Reshetnyak's majorizing theorem 299

3. Infinitesimal properties of CBA-spaces 300

3.1. Tangent cone 300

3.2. Scalar product and its concavity 300

3.3. Recognizing CBA-spaces 301

3.4. $\kappa$-convexity 302

3.5. Riemannian manifolds with boundary 304

3.6. Higher order properties 304

4. Local properties of CBA-spaces 305

4.1. Geometric dimension 305

4.2. Branch and singular points 306

4.3. Manifold points 306

4.4. Propagation from local to global 307

4.5. Busemann's G-spaces 308

5. Different types of convergence 308

6. Constructions 310

6.1. Gluing theorems 310

6.2. Warped products 312

$\begin{array}{ll}\text { 7. Gauss equation } & 314\end{array}$

*Supported by RFFI Grant 05-01-00939 and SNF Grant 20-668 33.01 
8. Extension results

8.1. Lipschitz extension property 315

8.2. Characterization of isometries 317

9. Rigidity results

10. 2-dimensional polyhedra

10.1. Singular edges are curves with bounded turn variation

10.2. Signed curvature measure and the Gauss-Bonnet formula for tame metrics

10.3. Gluing condition, characterization and approximation theorems

10.4. Rigidity due to Gauss-Bonnet 322

10.5. Metrics with bounded total curvature 323

References

\section{Motivation}

To motivate the study of spaces with curvature bounded from above (CBA for brevity) let us list some results which essentially use them.

1. The $p$-adic superrigidity of lattices in $\operatorname{Sp}(n, 1)$ and $\mathrm{F}_{4}$ was proven by developing the theory of harmonic maps into singular Non Positively Curved (NPC) spaces, e.g. Euclidean Bruhat-Tits buildings [59].

2 . For each $n \geq 5$, there are examples of closed topological $n$-manifolds $M^{n}$ with piecewise flat NPC metrics whose universal coverings $\widetilde{M}^{n}$ are not homeomorphic to $\mathbb{R}^{n}[\mathbf{5 5}]$. Furthermore, the interior of every compact contractible PL-manifold $C^{n}$, again $n \geq 5$, supports a complete metric $d$ of strictly negative curvature ([16], spines and metrization of polyhedra [29]). When $\partial C^{n}$ is not simply connected, there are geodesics of $d$ which are wild curves in $C^{n}$.

3. Solution of an old-standing problem concerning the existence of uniform estimates on the number of collisions in semi-dispersing billiards [50].

4. Examples of metric spaces with decent calculus, e.g. admitting Poincaré inequalities, for which all quasi-conformal automorphisms are quasisymmetric, with Hausdorff dimension not an integer. These spaces come out as ideal boundaries of some hyperbolic buildings $[43]$.

A beautiful survey on CBA spaces is [4], where also spaces with both lower and upper curvature bounds are discussed, see also [39]. That survey reflects the initial development of the subject up to 1986, when emphasis was placed on the comparison of angles and the angle excess of triangles rather than comparison of distances between sidepoints. Together with the background of CBA and CBB (curvature bounded from below) spaces, the notion of area, the Plateau problem and an isoperimetric inequality for a minimal 
surface are discussed. Significant part of that survey is dedicated to introducing a Riemannian structure on spaces with bi-sided curvature bounds under some additional assumptions, and to proving smoothness results.

Comprehensive introductions into the subject, especially for nonpositively curved spaces can be found in $[\mathbf{4 6}, \mathbf{2 1}, \mathbf{4 8}]$.

The global version of the CBA condition is the CAT property. We distinguish three major cases of $\mathrm{CAT}(\kappa)$-spaces with quite different flavors, results and approaches: $\kappa<0, \kappa=0$ and $\kappa>0$. CAT $(\kappa)$-spaces with $\kappa<0$ are Gromov hyperbolic and, conversely, by a result of M. Bonk and O. Schramm [51], every Gromov hyperbolic space (with a mild restriction) is roughly homothetic to a convex subset in $\mathrm{H}^{n}$. The most important examples are hyperbolic groups. All Hadamard spaces are CAT(0) and the tangent spaces of every CBA-space are Hadamard. Important examples of CAT(1)-spaces are the space of directions of a CBA-space and the Tits boundary at infinity $\partial_{T} X$ of a Hadamard space.

Each of the classes of CAT $(-1)$ and $\mathrm{CAT}(0)$ spaces deserves a separate treatment, especially with respect to rigidity results, which we only briefly touch in sect. 9. In this survey, we restrict ourself basically to those properties of $\mathrm{CBA}(\kappa)$-spaces which are independent of the sign of $\kappa$. In particular, we do not discuss (large scale) applications to non-positively curved spaces and to geometric group theory. We also regret that due to lack time and space we do not discuss an important paper [58].

Acknowledgment. We thank Stephanie Alexander for a number of valuable remarks. We also thank the referees for the many helpful comments and useful remarks. The first author is grateful to the University of Zürich for the support and the hospitality during his visit when a part of this survey has been written.

\section{Defining CBA}

2.1. CBA and CAT spaces. Throughout the paper, we use the notation $d(x, y)$ or $|x y|$ for the distance between points $x, y$ in a metric space.

Let $(X, d)$ be a metric space. The length of a (continuous) curve $\sigma:[a, b] \rightarrow X$ is given by

$$
L(\sigma):=\sup \sum_{j=1}^{k} d\left(\sigma\left(t_{j-1}\right), \sigma\left(t_{j}\right)\right) \in[0, \infty],
$$

where the supremum is taken over all positive integers $k$ and all subdivisions $a=t_{0} \leq t_{1} \leq \ldots \leq t_{k}=b$. Then

$$
d_{i}(x, y):=\inf \{L(\sigma): \sigma \text { is a curve from } x \text { to } y\}
$$

defines a semimetric on $X$, i.e. $d_{i}$ satisfies the axioms of a metric except that it may assume the value $\infty$ (one uses the convention that $r+\infty=\infty$ for 
$r \in[0, \infty])$. Slightly abusing the terminology, one calls $d_{i}$ the inner metric on $X$ induced by $d$. Note that $d_{i} \geq d$; if $d=d_{i}$ then $(X, d)$ is said to be an inner metric space. A curve $\sigma:[a, b] \rightarrow X$ is called minimizing or shortest if $L(\sigma)=d(\sigma(a), \sigma(b))$. Then $\sigma$ is said to be a minimizing geodesic if it additionally has constant speed, i.e. there exists $s \geq 0$ such that $L(\sigma \mid[a, t])=$ $s(t-a)$ for all $t \in[a, b]$. A metric space $X$ is called locally geodesic if every $p \in X$ possesses a neighborhood $U$ such that for all $x, y \in U$ there exists a minimizing geodesic in $X$ from $x$ to $y . X$ is called a geodesic space if this holds for $U=X$.

For $\kappa \in \mathbb{R}$, let $M_{\kappa}$ be the model 2-space of constant curvature $\kappa$, define

$$
D_{\kappa}:=\operatorname{diam} M_{\kappa}= \begin{cases}\pi / \sqrt{\kappa} & \text { for } \kappa>0 \\ \infty & \text { for } \kappa \leq 0\end{cases}
$$

A triangle in $X$ is a triple $\Delta=\left(\sigma_{1}, \sigma_{2}, \sigma_{3}\right)$ of minimizing geodesics $\sigma_{i}:\left[a_{i}, b_{i}\right] \rightarrow X$ whose endpoints match as usual. Assume that $\Delta$ has perimeter

$$
P(\Delta):=L\left(\sigma_{1}\right)+L\left(\sigma_{2}\right)+L\left(\sigma_{3}\right)<2 D_{\kappa} .
$$

Then there exists a comparison triangle $\Delta^{\kappa}$ for $\Delta$ in $M_{\kappa}$ which is unique up to isometry, namely, a triple of geodesic segments $\sigma_{i}^{\kappa}:\left[a_{i}, b_{i}\right] \rightarrow M_{\kappa}$ such that $L\left(\sigma_{i}^{\kappa}\right)=L\left(\sigma_{i}\right)$ for $i=1,2,3$, and such that the endpoints of $\sigma_{1}^{\kappa}, \sigma_{2}^{\kappa}, \sigma_{3}^{\kappa}$ match in the same way as those of $\sigma_{1}, \sigma_{2}, \sigma_{3}$. Then $\Delta$ is said to be $\kappa$-thin if $d\left(\sigma_{i}(s), \sigma_{j}(t)\right) \leq d\left(\sigma_{i}^{\kappa}(s), \sigma_{j}^{\kappa}(t)\right)$ respectively, whenever $i, j \in\{1,2,3\}, s \in$ $\left[a_{i}, b_{i}\right]$, and $t \in\left[a_{j}, b_{j}\right]$.

Definition 2.1 $(\mathrm{CBA}(\kappa))$. A metric space $X$ is said to have curvature $\leq \kappa$, or it is called a $\mathrm{CBA}(\kappa)$-space, if it is locally geodesic and every $p \in X$ possesses a neighborhood $U$ such that all triangles in $X$ with vertices in $U$ and perimeter $<2 D_{\kappa}$ are $\kappa$-thin.

The global version of this definition is the following

Definition $2.2(\mathrm{CAT}(\kappa))$. A metric space $X$ is called a $\mathrm{CAT}(\kappa)$ space if it is geodesic and all triangles in $X$ of perimeter $<2 D_{\kappa}$ are $\kappa$-thin.

It follows that for all $x, y \in X$ with $d(x, y)<D_{\kappa}$ there is a unique minimizing geodesic $\sigma:[0,1] \rightarrow X$ from $x$ to $y$, and all metric balls in $X$ with radius $<D_{\kappa} / 2$ are strongly convex. Note that $M_{\kappa}$ is a $\operatorname{CAT}(\kappa)$ space.

In general, the convexity radius $r(x)$ at $x \in X$ of a CBA-space $X$ is the supremum of $r>0$ such that the ball $B_{r}(x)$ is strictly convex. Taking the infimum over all $x \in X$, we obtain the convexity radius of $X$.

There is another characterization of CBA-spaces via the Lipschitz extension property which is useful in some cases. We say that a metric space $U$ is $\operatorname{CAT}(\kappa)$ if for every triple $S=\{\bar{x}, \bar{y}, \bar{z}\} \subset M_{\kappa}$ with perimeter $<2 D_{\kappa}$ and every isometric $f: S \rightarrow U$, there is a 1-Lip extension $\{\bar{y}, \bar{t}, \bar{z}\} \rightarrow U$ of $f \mid\{\bar{y}, \bar{z}\}$, where $\bar{t} \in \overline{y z}$ is the midpoint, and every such extension defines a 
1-Lip extension $\bar{f}: S \cup\{\bar{t}\} \rightarrow U$ of $f$. In this case, a triple $f(S)=\{x, y, z\}$ is said to be $\kappa$-thin. A metric space $X$ is $\operatorname{CBA}(\kappa)$ if it is $\operatorname{CAT}(\kappa)$ locally.

This definition is actually equivalent to the definition above under the assumption that $X$ is complete.

REMARK 2.3. In the definition of a CAT $(\kappa)$-space, it suffices to compare the medians of triangles. On the other hand, comparing the midlines instead of the medians leads to Busemann's condition of nonpositive curvature (for $\kappa=0)$. The corresponding class of spaces is much larger than $\mathrm{CBA}(0)$ and includes, in particular, linear normed spaces. The basic distinction is that $\mathrm{CBA}(\kappa)$ implies the existence of angles whereas Busemann's NPC does not. Actually the Busemann NPC condition plus the existence of angles is equivalent to $\mathrm{CBA}(0)$.

REMARK 2.4. One can redefine the CAT $(\kappa)$-condition in an equivalent way saying that for each $\varepsilon>0,0<P<2 D_{\kappa}$ there exists $\delta>0$ such that for every $(1, \delta)$-quasi-isometric $f: S \rightarrow U$ with perimeter of $S$ bounded by $P$, every $(1, \delta)$-quasi-isometric extension $\{\bar{y}, \bar{t}, \bar{z}\} \rightarrow U$ of $f \mid\{\bar{y}, \bar{z}\}$ with the midpoint $\bar{t} \in \overline{y z}$ defines a $(1, \varepsilon)$-quasi-isometric extension $\bar{f}: S \cup\{\bar{t}\} \rightarrow U$ of $f$. This stabilized definition is useful for proving that the CBA-condition behaves well under limiting operations.

A very important class of CBA spaces is the class of Hadamard spaces.

Definition 2.5 (Hadamard space). A Hadamard space is a complete $\mathrm{CAT}(0)$ space.

2.2. Reshetnyak's majorizing theorem. There is a broad generalization of the defining property of a $\mathrm{CAT}(\kappa)$ space which is an important tool for the study of CBA-spaces [100].

TheOREM 2.6 (RMT). Let $\gamma$ be a closed curve of length $<2 D_{\kappa}$ in a $\mathrm{CAT}(\kappa)$ space $M$. Then there is a closed curve $\widetilde{\gamma}$ which is the boundary of a convex region $D$ in $M_{\kappa}$ and a distance non-increasing map $\phi: D \rightarrow M$ such that the restriction of $\phi$ to $\widetilde{\gamma}$ is an arclength-preserving map onto $\gamma$.

Taking $\gamma$ to be a triangle, we get back the defining property of a $\operatorname{CAT}(\kappa)$ space because for a geodesic subarc of $\gamma$, the corresponding subarc of $\widetilde{\gamma}$ is also a geodesic segment.

It is very surprising how far one can get by starting with the $\mathrm{CBA}(\kappa)$ condition. For a complete $X$, this condition implies the local existence and uniqueness of geodesics, the existence and comparison of angles, the propagation from local to global comparison, the infinitesimal theory etc. The basic device to extract various properties from the definition is the following.

LEMMA 2.7. Assume that $x y t$, xtz are $\kappa$-thin triangles, where $t \in y z$. Then, if the perimeter of $x y z$ is less than $2 D_{\kappa}$, the triangle $x y z$ is also $\kappa$-thin. 


\section{Infinitesimal properties of CBA-spaces}

3.1. Tangent cone. Let $\Sigma$ be a metric space with diam $\Sigma \leq \pi$. The Euclidean cone $C(\Sigma)$ over $\Sigma$ is defined as follows. The underlying set will be $\Sigma \times[0, \infty) / \Sigma \times\{0\}$. Given $\sigma_{1}, \sigma_{2} \in \Sigma$, we consider embeddings $\rho:\left\{\sigma_{1}, \sigma_{2}\right\} \times$ $[0, \infty) \rightarrow \mathbb{R}^{2}$ such that $\left|\rho\left(\sigma_{i}, t\right)\right|=|t|$ and $\angle_{0}\left(\rho\left(\sigma_{1}, t_{1}\right), \rho\left(\sigma_{2}, t_{2}\right)\right)=\left|\sigma_{1} \sigma_{2}\right|$, and we equip $C(\Sigma)$ with the unique metric for which these embeddings are isometric. The space $C(\Sigma)$ is $\operatorname{CAT}(0)$ if and only if $\Sigma$ is $\operatorname{CAT}(1)$.

Given a $\operatorname{CBA}(\kappa)$-space $X, x \in X$, we let $\Sigma_{x} X$ be the direction space at $x$ that is the (metric completion of the) set of equivalence classes of geodesic segments with initial point $x$ (two such segments are equivalent if they have zero angle at $x$ ). The tangent cone of $X$ at $x$, denoted by $T_{x} X$, is the Euclidean cone $C\left(\Sigma_{x} X\right)$.

THEOREM 3.1. If $X$ is locally compact and geodesically complete, then $T_{x} X$ is a Hausdorff-Gromov limit of blow-ups $\frac{1}{\varepsilon}(X, x)$ as $\varepsilon \rightarrow 0$. In general, the $\mathrm{CBA}(\varepsilon \kappa)$-spaces $\frac{1}{\varepsilon}(X, x)$ converge to $T_{x} X$ on finite subsets. Thus the tangent cone $T_{x} X$ of a CBA-space $X$ is a CAT(0)-space at every $x \in X$; consequently the direction space $\Sigma_{x} X$ is a CAT(1)-space.

(The last fact is due to I. Nikolaev, $[\mathbf{9 0}]$ ). The proof is straightforward, based on the existence of angles and uses the stabilized definition of the CAT $(\kappa)$-condition. The direction spaces of CBA-spaces were studied in [33, $49,66,73,74,90,93]$.

3.2. Scalar product and its concavity. We use the notation $v=r \sigma$ for $v=(\sigma, r) \in T_{x} X$. Given $v=r \sigma, v^{\prime}=r^{\prime} \sigma^{\prime} \in T_{x} X$ one defines their scalar product

$$
\left\langle v, v^{\prime}\right\rangle:=\frac{1}{2}\left(r^{2}+r^{\prime 2}-d_{x}\left(v, v^{\prime}\right)^{2}\right)
$$

The CAT(0)-property of $T_{x} X$ implies the concavity of the scalar product: whenever $\gamma:[0,1] \rightarrow T_{x} X$ is a geodesic and $w \in T_{x} X$, then

$$
\langle\gamma(t), w\rangle \geq(1-t)\langle\gamma(0), w\rangle+t\langle\gamma(1), w\rangle
$$

for all $t \in[0,1]$. By iterating this inequality one obtains the following.

Proposition 3.2. Let $C \subset T_{x} X$ be the convex hull of a finite set $\left\{u_{1}, \ldots\right.$, $\left.u_{k}\right\}$. Then for every $v \in C$ there exist $\mu_{1}, \ldots, \mu_{k} \geq 0$ with $\sum_{i} \mu_{i}=1$ such that

$$
\sum_{i} \mu_{i}\left\langle u_{i}, w\right\rangle \leq\langle v, w\rangle
$$

for all $w \in T_{x} X$.

This is due to U. Lang and V. Schroeder, [73], who together with B. Pavlović, [75], have used it to establish a remarkable Lipschitz extension property of Hadamard spaces, see sect. 8.1. 
3.3. Recognizing CBA-spaces. We give a fundamental example of a space which is not CBA. This is the Euclidean cone $X$ over a circle of length $<2 \pi$. Clearly, $X$ contains arbitrarily short geodesic bigons (which live near the vertex), thus it cannot be a CBA-space.

Basic examples of $\mathrm{CBA}(\kappa)$-spaces are simplicial complexes obtained by gluing together simplices of constant curvature $\kappa$. In such a complex, the link of each simplex has itself the structure of a complex built out of spherical simplices (the case $\kappa=1$ ). It turns out that (see $[\mathbf{2 0}]$ ):

Theorem 3.3. The original complex will be $\mathrm{CBA}(\kappa)$ if and only if the link of every simplex is (globally) CAT(1). This is equivalent to saying that the link of each simplex should contain no closed geodesic of length strictly less than $2 \pi$.

Closely related is the following result due to V. Berestovskii [29].

THEOREM 3.4. Any simplicial complex admits a piecewise spherical CAT (1)-metric.

For the proof, one should take the barycentric subdivision and introduce the metric in which every simplex of the subdivision is isometric to the standard spherical one of the same dimension.

This result has several important applications, among them are examples of CBA(1)-spaces homeomorphic to $n$-manifolds $(n \geq 5)$ which contain points where the direction space is not homeomorphic to a manifold, see [31] (this settles in the negative a question of A. Aleksandrov). Take a homological Poincaré sphere $\Sigma^{3}$ and let $X=S^{1}\left(S^{1}\left(\Sigma^{3}\right)\right)$ be its double suspension. Its known that $X$ is homeomorphic to $S^{5}$, whereas $Y=S^{1}\left(\Sigma^{3}\right)$ is not a manifold. Starting with a triangulation of $\Sigma^{3}$ one introduces a CBA(1)-metric on $X$ such that the direction space at some point is $Y$. In dimension $n=3,4$ the problem seems to be open (and related to the Poincaré conjecture for $n=4)$. It follows from [49, Prop. 3.12] that the space of directions $\Sigma_{x}$ at every point is weakly homotopy equivalent to $S^{n-1}$. We do not know, even for $n=3$, whether $\Sigma_{x}$ is a finite 2-polyhedron. On the other hand, there are simple examples of finite 2-polyhedra with complete metrics of upper bounded curvature and with extendable geodesics that are homotopy equivalent, but not homeomorphic to the sphere $S^{2}$.

The paper by P. Thurston [104] has some relation to this problem. It is shown there that for arbitrarily small $r>0$, the metric sphere $S_{r}$ of radius $r$ around a point in a topological $n$-manifold with CBA-metric is homeomorphic to $S^{2}$ for $n=3$ and it is homeomorphic to a closed 3-manifold for $n=4$. However, it is not sufficient for the solution of the Alexandrov problem even for $n=3$ because we can only say that $S_{r}$ converge to the link at the respective point by Hausdorff-Gromov. But there are easy examples of sequences of metric 2-spheres which Hausdorff-Gromov converge e.g. to a 2-disc.

In this respect, we would like to mention some results of a sphere theorem type. A (nontrivial) geodesic space $X$ is geodesically complete if every 
nontrivial geodesic $\gamma: J \rightarrow X, J \subset \mathbb{R}$ can be extended as a locally isometric embedding to the whole real line $\mathbb{R}$. Assume that $0<\mathcal{H}^{n}\left(B_{r}(x)\right)<\infty$, $n \in \mathbb{N}$, for every point $x \in X$ of a compact, geodesically complete CAT(1)space $X$ and for all sufficiently small $r>0$, where $\mathcal{H}^{n}$ is the $n$-dimensional Hausdorff measure.

It is proven in $[88]$ that then $\mathcal{H}^{n}(X) \geq \operatorname{vol} S^{n}$ for the unit sphere $S^{n} \subset \mathbb{R}^{n+1}$. Moreover, if in addition $\mathcal{H}^{n}(X)<\operatorname{vol} S^{n}+\varepsilon_{n}$ for some $\varepsilon_{n}>0$ depending only on $n \geq 1$, then $X$ is bi-Lipschitz homeomorphic to $S^{n}$ with bi-Lipschitz constants close to 1 .

In dimension $n=2$ there is a much better result [89]: if

$$
\mathcal{H}^{2}(X)<6 \pi\left(=3 / 2 \operatorname{vol} S^{2}\right)
$$

then, under the conditions above, $X$ is homeomorphic to $S^{2}$. This result is optimal because the union $X$ of $S^{2}$ and a hemisphere $S_{+}^{2}$ along an equator is not homeomorphic to $S^{2}$ while $\mathcal{H}^{2}(X)=3 / 2 \operatorname{vol} S^{2}$.

3.4. $\kappa$-convexity. Let $\mathcal{F}_{\kappa}$ denote the family of solutions of the differential equation $f^{\prime \prime}+\kappa f=0, \kappa \in \mathbb{R}$. We say that a continuous function $f: X \rightarrow \mathbb{R}$ on a geodesic metric space $X$ is $\kappa$-convex if its restriction to every unit speed geodesic satisfies the differential inequality

$$
f^{\prime \prime}+\kappa f \geq 0
$$

in the barrier sense. This means that $f \leq g$ if $g \in \mathcal{F}_{\kappa}$ coincides with $f$ at the end points of a sufficiently short subsegment. Thus $\mathcal{F}_{0}$-convexity is usual convexity.

A real function $F$ along a geodesic $\gamma$ in $X$ is called a normal Jacobi field length if there is a sequence of geodesics $\gamma_{i}$ and a sequence of positive numbers $u_{i}$ approaching 0 for which

$$
F(t)=\lim u_{i}^{-1}\left|\gamma_{i}(t) \gamma(t)\right|,
$$

where all $\gamma_{i}$ and $\gamma$ are arclength parameterized by $[0, l]$, and $\left|\gamma_{i}(t) \gamma(t)\right|=$ $\operatorname{dist}\left(\gamma_{i}(t), \gamma\right)+o\left(u_{i}\right)$ for all $t \in[0, l]$. In the case of Riemannian manifolds with boundary, this notion is studied in detail in $[\mathbf{1 3}, \mathbf{1 4}]$ together with the notion of Jacobi field direction. In particular, existence and regularity results are obtained.

For this discussion, we do not need the notion of Jacobi field direction.

Proposition 3.5. Assume that a geodesic metric space $X$ is $\operatorname{CBA}(\kappa)$ for some $\kappa \in \mathbb{R}$. Then every normal Jacobi field length along every unit speed geodesic is $\kappa$-convex.

This easily follows from RMT applied to geodesic quadrilaterals $\gamma(0) \gamma(l)$ $\gamma_{i}(l) \gamma_{i}(0)$, and properties of geodesics in the model space $M_{\kappa}$, see $[\mathbf{1 4}]$. In the case $\kappa \geq 0$, the condition to be normal, i.e. that $\left|\gamma_{i}(t) \gamma(t)\right|=\operatorname{dist}\left(\gamma_{i}(t), \gamma\right)+$ $o\left(u_{i}\right)$, can be omitted without violating the conclusion of the proposition. 
The converse is proved in a number of cases, see $[\mathbf{1 4}, \mathbf{1 1}]$, and the argument requires the following (local) properties of a geodesic metric space $X$ :

- every point of $X$ has a neighborhood in which any geodesic variation whose endpoint curves are Lipschitz is itself Lipschitz;

- the first variation formula;

- existence of the Jacobi field lengths and their splitting into the normal and tangential components, where the last one is linear.

For example, these conditions are obviously fulfilled in the case $X$ is a Riemannian manifold (without boundary). It is more delicate to show that they are also fulfilled for a Riemannian manifold with nonempty boundary, see $[\mathbf{1 3}, \mathbf{1 4}]$ and the next section. Another important case, namely when $X$ is CBA, is discussed in [11], see sect. 7 .

Proposition 3.6. Assume that a geodesic metric space $X$ possesses the properties above. If every normal Jacobi field length is $\kappa$-convex, then $X$ is $\operatorname{CBA}(\kappa)$.

This proposition implies in particular that every Riemannian manifold with sectional curvatures $\leq \kappa$ is $\mathrm{CBA}(\kappa)$.

The idea is to prove the angle comparison condition for any sufficiently small triangle $p q r \subset X$. To this end, a "development" argument of the kind introduced by Alexandrov [2] is used as follows, compare [14]. Connect $p$ with every point of $q r$ by the minimizer and develop this variation as a map into $M_{\kappa}$ which also has the form of a cone over a curve. Two of the developped sides, $\overline{p q}$ and $\overline{p r}$, are geodesics of the same length as $p q$ and $p r$ respectively and the third side, $\overline{q r}$, is a curve of the same length as $q r$. It suffices to show that the angle $\bar{\theta}$ at $\bar{p}$ swept out by the comparison cone is at least the angle $\theta$ at $p$ in $p q r$. Choosing points $x \in p q, y \in p r$ sufficiently close to and different from $p$, connect the comparison points $\bar{x} \in \overline{p q}, \bar{y} \in \overline{p r}$ by the shortest path $\bar{\tau}$ within the comparison cone. Lifting $\bar{\tau}$ to the curve $\tau$ connecting $x$ and $y$ in the initial cove over $q r$, we prove that the length of $\tau$ is not greater than that of $\bar{\tau}$ by representing the speed of $\tau$ as the value of a Jacobi field length along appropriate $p z, z \in q r$, decomposing it into the normal and tangent components and using the $\kappa$-convexity of the normal component while the tangent component coincides with that in the comparison cone. This implies the required $\theta \leq \bar{\theta}$.

Proposition 3.6 is typically used to establish a sharp $\mathrm{CBA}(\kappa)$ condition, for example when it is already known that a given space $X$ is $\mathrm{CBA}$, see $[\mathbf{6 8}$, $\mathbf{7 0}, \mathbf{1 1}]$. Yet, it is interesting to find general and effective conditions which would imply the conditions of Proposition 3.6. The notion of a geometric space, introduced in $[\mathbf{7 8}]$, seems to be suggestive in this respect.

According to [78], a proper geodesic space $X$ is geometric if the following holds:

- for every $x \in X$ the union of all geodesics starting at $x$ contains a neighborhood of $X$ and for any two such geodesics $\gamma, \gamma^{\prime}$ the limit 
$\lim _{t \rightarrow 0}\left|\gamma(t) \gamma^{\prime}(s t)\right| / t$ exists for all $s>0$. Moreover, we require the following uniformity condition in the limit above: for each $\varepsilon>0$ there is $\rho>0$ such that $\left|\gamma(t) \gamma^{\prime}(t)\right| \leq \varepsilon t$ for all positive $t<\rho$ and $\gamma, \gamma^{\prime}$ whose directions at $x$ are $\rho$-close to each other;

- each tangent space $T_{x} X, x \in X$, is uniformly convex and smooth;

- geodesics vary smoothly in $X$.

For precise definitions we refer to [78]. The following classes of spaces are geometric: CBA and $\mathrm{CBB}$ (curvature bounded below) spaces; extremal subsets in $\mathrm{CBB}$ spaces [96]; surfaces with bounded total curvature (see sect. 10.5); Hölder continuous Riemannian manifolds; sufficiently convex and smooth Finsler manifolds; subsets of positive reach in CBA spaces (see sect. 7). The class of geometric spaces is closed under natural metric operations and the first variation formula holds for the geometric spaces.

3.5. Riemannian manifolds with boundary. The following important result is obtained in $[\mathbf{1 4}]$.

TheOREM 3.7. Let $M$ be a Riemannian manifold with boundary B. Then the following two conditions are equivalent:

(1) $M$ is $\operatorname{CBA}(\kappa)$.

(2) The sectional curvatures of the interior of $M$ and the outward sectional curvatures of the boundary $B$ do not exceed $\kappa$ (where an outward sectional curvature of $B$ is one that corresponds to a tangent section all of whose normal curvature vectors point outward).

A characteristic difficulty lies in the possibility of unbounded switching behavior, which may, for example, produce Cantor coincidence sets between a geodesic and the boundary.

This theorem is proven by establishing the equivalence of conditions 1 and 2 with a third condition, namely, the $\kappa$-convexity of normal Jacobi field lengths. The most difficult part of Theorem 3.7 is to prove that condition 2 implies $\kappa$-convexity of normal Jacobi field lengths, especially at points of a geodesic $\gamma \subset M$ lying in the boundary where the acceleration exists and vanishes.

As an application, the following Hadamard-Cartan theorem for manifolds with boundary is obtained in $[\mathbf{1 4}]$.

COROLlary 3.8. If for a simply connected, complete Riemannian manifold with boundary, the sectional curvatures of the interior and the outward sectional curvatures of the boundary are nonpositive, then any two points are joined by a unique geodesic, and the distance between any two geodesics is convex.

3.6. Higher order properties. On the unit tangent bundle $U M$ of every Riemannian manifold, there is a natural Riemannian metric, called 
the Sasaki metric, which is defined via the Levi-Civita parallel transport, $[\mathbf{1 0 1}, \mathbf{1 0 2}]$. An attempt to define an analog of the Sasaki metric or, more precisely, of the notion of angle between two directions, possibly based at different points, is made in [40] for general CBA-spaces. The approach is based on the notions of quadrilateral cosine and sine, where the later is defined via the former. The definition of the quadrilateral sine is rather involved and for general CBA-space it is hard to prove anything useful about that notion, see $[\mathbf{4 0}]$. In the case of spaces with more regularity, one can achieve more advances and more interesting results involving higher order properties, see $[\mathbf{9 1}, \mathbf{9 2}]$.

\section{Local properties of CBA-spaces}

4.1. Geometric dimension. One defines the geometric dimension of CBA spaces to be the smallest function (taking values in $\mathbb{N} \cup \infty$ ) on the class of CBA spaces such that

(1) $\operatorname{GeomDim}(X)=0$ if $X$ is discrete;

(2) $\operatorname{GeomDim}(X) \geq 1+\operatorname{GeomDim}\left(\Sigma_{x} X\right)$ for every $x \in X$.

In other words, to find the geometric dimension of a CBA-space we look for the largest number of times that we can pass to spaces of directions without getting the empty set. This notion and the related results are due to B. Kleiner, [65].

TheOREM 4.1. For every $C B A$-space $X$ we have

$$
\operatorname{GeomDim}(X)=\sup \{\operatorname{TopDim}(K): K \subset X \text { is compact }\} \text {, }
$$

where TopDim is the topological dimension.

Let $X$ be a CBA space with $\operatorname{GeomDim}(X)=n<\infty$. Then $\sup \{k$ : $\forall \varepsilon>0 \exists(1+\varepsilon)$ - bilipschitz embedding $U \rightarrow X$ of an open $\left.U \subset \mathbb{R}^{k}\right\}=n$.

Let $X$ be a locally compact Hadamard space on which $\operatorname{Isom}(X)$ acts cocompactly. Then $\sup \left\{k:\right.$ There is an isometric embedding $\left.\mathbb{R}^{k} \rightarrow X\right\}=$ $1+\operatorname{GeomDim}\left(\partial_{T} X\right)$.

Here $\partial_{T} X$ is the boundary at infinity of $X$ equipped with the Tits metric. Actually, a number of other properties related to $\operatorname{GeomDim}(X)$ are proved in $[\mathbf{6 5}]$. Here we have listed only the most important ones.

A key ingredient in the proofs is the notion of a barycentric simplex. For $z=\left(z_{0}, \ldots, z_{n}\right) \subset X$ (with sufficiently small diameter if $\kappa>0$ ), the barycentric simplex determined by $z$ is the singular simplex $\sigma_{z}: \Delta_{n} \rightarrow$ $X$ which maps each $\alpha=\left(\alpha_{0}, \ldots, \alpha_{n}\right) \in \Delta_{n}$ to the unique minimum of the uniformly convex function $\phi_{\alpha}=\sum \alpha_{i} \operatorname{dist}\left(z_{i}, \cdot\right)^{2}$. Barycentric simplices are Lipschitz and possess the following remarkable property.

If $x \in \sigma_{z}\left(\Delta_{n}\right) \backslash \sigma_{z}\left(\partial \Delta_{n}\right)$, then $\operatorname{GeomDim}\left(\Sigma_{x}\right) \geq n-1$.

For the proof, one considers the differential $g_{\alpha}=d \phi_{\alpha}=\sum \alpha_{i} f_{i}: T_{x} X \rightarrow$ $\mathbb{R}$. It turns out that for $\alpha \in \partial \Delta_{n}$, its restriction to the unit sphere $\Sigma_{x} X \subset$ 
$T_{x} X$ possesses a unique minimum and thus defines a Lipschitz $\sigma_{f}: \partial \Delta_{n} \rightarrow$ $\Sigma_{x} X$ which is a nondegenerate $(n-1)$-chain of barycentric simplices. Arguing by induction, one concludes that $\operatorname{GeomDim}\left(\Sigma_{x} X\right) \geq n-1$.

This leads to the estimate $\operatorname{TopDim}(K) \leq \operatorname{GeomDim}(X)$ for compact subsets $K \subset X$, and using the nondegenerate part $\sigma_{z}\left(\Delta_{n}\right) \backslash \sigma_{z}\left(\partial \Delta_{n}\right)$, one obtains bilipschitz embeddings of open sets $U \subset \mathbb{R}^{n}$ into $X$.

Though it is not stated explicitly, the existence of bilipschitz embeddings $U \subset \mathbb{R}^{n} \rightarrow X$ should imply (an extension of) the Rademacher-Stepanoff theorem on the differentiability of Lipschitz functions to CBA-spaces, cf. $[67],[54]$.

4.2. Branch and singular points. Let $X$ be a locally compact, geodesically complete CBA-space. A point $x \in X$ is said to be regular, if the direction space $\Sigma_{x} X$ is isometric to the unit sphere $S^{n-1}$ for some $n \in \mathbb{N}$, while $\mathcal{H}^{n}\left(B_{r}(x)\right)<\infty$ for some $r>0$. A point $x \in X$ that is not regular is called singular.

Let $x \in X, \delta>0$. Following [94], we say that $v \in \Sigma_{x} X$ is a $\delta$-branch direction, if diam $B_{v} \geq \delta$, where $B_{v} \subset \Sigma_{x} X$ consists of all directions forming the (maximal possible) angle $\pi$ with $v$. Furthermore, $y \in X$ is called a $\delta$ branch point of $x$, if $v_{y x} \in \Sigma_{y} X$ is a $\delta$-branch direction, where the direction $v_{y x}$ is tangent to some geodesic segment $y x$. We denote by $S_{x, \delta}$ the set of all $\delta$-branch points of $x$ and by $S_{\delta}$ the set of all $\delta$-branch points, $S_{\delta}=\cup_{x \in X} S_{x, \delta}$. Note that if $x \in S_{\delta}$ then $\Sigma_{x} X$ is not isometric to $S^{n-1}$ for any $n \in \mathbb{N}$. In particular, it follows that $S_{\delta}$ consists of singular points.

The following result $[\mathbf{9 4}, \mathbf{8 8}]$ shows the abundance of regular points.

TheOREM 4.2. Assume that $\mathcal{H}^{n}\left(B_{r}(x)\right)<\infty$ for some $n \in \mathbb{N}, x \in X$ and a sufficiently small $r>0$. Then $\mathcal{H}^{n}\left(S_{x, \delta} \cap B_{r}(x)\right)=0$ for any $\delta>0$. Moreover, $\mathcal{H}^{n}\left(S_{\delta} \cap B_{r}(x)\right)=0$ and $\mathcal{H}^{n}\left(A_{n} \cap B_{r}(x)\right)=0$, where $A_{n} \subset X$ consists of all $x$ with $\Sigma_{x} X$ not isometric to $S^{n-1}$.

We conclude that if $0<\mathcal{H}^{n}\left(B_{r}(x)\right)<\infty$ for all sufficiently small $r>0$, then $\mathcal{H}^{n}$-almost every point of every such ball is regular.

4.3. Manifold points. A manifold point in a metric space $X$ is a point with a neighborhood homeomorphic to an open subset in some $\mathbb{R}^{n}$. The following result is due to B. Kleiner. For simplicity, we give a qualitative version.

THEOREM 4.3. If a metric ball $B_{r}(x)$ in a geodesically complete CBAspace $X$ is sufficiently close in the Hausdorff-Gromov metric (see sect. 5) to the ball $B_{r}(0) \subset \mathbb{R}^{n}$ of the same sufficiently small radius $r>0$, then $a$ smaller concentric ball $B_{\rho}(x) \subset B_{r}(x)$ with $\rho \ll r$ is bi-Lipschitz homeomorphic to an open subset of $\mathbb{R}^{n}$. 
For the proof see $[49, \S 3]$, a quantitative version can be found in [88]. This theorem is similar to [47, Theorem 5.4] for CBB-spaces, and the argument follows a similar line of reasoning by proving the existence of a distance frame or strainer and studying the associated distance map into $\mathbb{R}^{n}$. The essential distinction to the CBB case is only that a lower estimate for angles is based on the extendability of geodesics. For applications to structure results for CBA-metrics on 2-polyhedra see [49] and sect. 10, for applications to a volume convergence theorem see $[\mathbf{8 8}]$ and the end of sect. 5 .

If every point of $X$ is a manifold point, then $X$ is called a CBA-manifold. Even locally CBA-manifold can differ significantly from Riemannian manifolds, as the examples of CBA-manifolds whose space of directions $\Sigma_{x}$ is not a manifold (section 3.3) show. For every $n \geq 5$ M. Davis and T. Januszkiewicz [55] constructed CBA(0)-manifolds $M^{n}$ whose universal covering space $X=$ $\widetilde{M}^{n}$ is not simply connected at infinity, in particular not homeomorphic to $\mathbb{R}^{n}$. P. Thurston [104] however showed, that a 4-dimensional CAT(0)manifold $X^{4}$ which possesses a tame point, is homeomorphic to $\mathbb{R}^{4}$. Here a point $x \in X$ is called tame, if for all $r>0$ the distance sphere $S_{r}(x)$ is a closed manifold.

4.4. Propagation from local to global. A remarkable property of the comparison conditions CBA as well as CBB is that they propagate from local to global. However, there is a fundamental distinction between CBA and CBB: in any $\operatorname{CBB}(\kappa)$ space every geodesic triangle satisfies the angle comparison with $M_{\kappa}$ (Toponogov's theorem), whereas for CBA spaces this is not the case. This is a major source of problems for proving or checking the CBA-condition.

A geodesic $\gamma:[0,1] \rightarrow X$ has no conjugate points, if for some neighborhood $U$ of $\gamma$ (in the space of maps $[0,1] \rightarrow X$ with the compact-open topology) the map $p: U \rightarrow X \times X, p(\sigma)=(\sigma(0), \sigma(1))$ is a homeomorphism on the neighborhood $V=p(U)$ of $(\gamma(0), \gamma(1))$. We have [5] the following:

TheOREM 4.4. Every geodesic $\gamma$ of length $L(\gamma)<D_{\kappa}$ in a complete $\mathrm{CBA}(\kappa)$-space $X$ has no conjugate points. Moreover, every narrow triangle with two sides sufficiently close to $\gamma$ is $\kappa$-thin.

The main ingredient of the proof is the following middle-third construction. For geodesics $\gamma, \sigma, \gamma^{\prime}, \sigma^{\prime}:[0,1] \rightarrow X$ such that $\gamma^{\prime}(0)=\gamma(0)$, $\gamma^{\prime}(1)=\sigma(1 / 2), \sigma^{\prime}(0)=\gamma(1 / 2), \sigma^{\prime}(1)=\sigma(1)$ we denote $\left(\gamma^{\prime}, \sigma^{\prime}\right)=\Lambda(\gamma, \sigma)$ and put $\mu(\gamma, \sigma)=\max \{|\gamma(1 / 2) \sigma(0)|,|\gamma(1) \sigma(1 / 2)|\}$.

For every $0<P<\frac{2}{3} D_{\kappa}$ there exist $\delta=\delta(P), \lambda=\lambda(P), \delta>0,0<\lambda<1$ such that if for pairs of geodesics $(\gamma, \sigma),\left(\gamma^{\prime}, \sigma^{\prime}\right)=\Lambda(\gamma, \sigma)$ in a metric space $X$ the triangles $\Delta$ with sides $\gamma, \gamma^{\prime}, \gamma(1) \gamma^{\prime}(1)$ and $\Delta^{\prime}$ with sides $\sigma, \sigma^{\prime}, \sigma(0) \sigma^{\prime}(0)$ are $\kappa$-thin and $P(\Delta), P\left(\Delta^{\prime}\right) \leq P, \mu(\gamma, \sigma)<\delta$ then

$$
\mu\left(\gamma^{\prime}, \sigma^{\prime}\right) \leq \lambda \mu(\gamma, \sigma) .
$$


Applied to the case $\kappa \leq 0$ this leads to an extension of the classical Hadamard-Cartan theorem to Hadamard spaces.

TheOREM 4.5. For every $\kappa \leq 0$, every complete simply connected CBA $(\kappa)$-space satisfies $\mathrm{CAT}(\kappa)$.

There is a standard trap in the proof: if one has a space in which a geodesic between any two points is unique then it is natural to suppose that the geodesics vary continuously with their ends. This is indeed the case for the locally compact $\mathrm{CBA}(\kappa)$-spaces. However, in general, this is not true. One can observe this effect by looking at the cartwheel. As an example (due to W. Ballmann) one can take the metric completion $X$ of $B=\cup_{n} B_{n}$ where $B_{1}$ is the circle of length 3 , and $B_{n+1}$ is obtained from $B_{n}$ by connecting every $x, y \in B_{n}$ with $\operatorname{dist}(x, y)>1$ by a segment of length 1 . Then $X$ is a complete $\operatorname{CBA}(\kappa)$-space for each $\kappa \in \mathbb{R}$ such that every two points from the dense subset $B$ are connected by a unique geodesic. However, geodesics in $X$ do not vary continuously with their ends.

4.5. Busemann's G-spaces. A G-space of Busemann is a locally compact, complete, inner metric space in which geodesics are not overlapping and locally extendable (geodesics are not overlapping if whenever two of them, $\gamma$ and $\gamma^{\prime}$, have an open common interval, their union $\gamma \cup \gamma^{\prime}$ again supports a geodesics). The following result due to V. Berestovskii [33] describes G-spaces which are CBA.

TheOrem 4.6. Every CBA G-space of Busemann is a Riemannian $C^{0}$ manifold. The components of the metric tensor are continuous w.r.t. distance coordinates. Every two distance coordinates maps are $C^{1}$-compatible.

REMARK 4.7. A similar result holds true for CBB G-spaces, moreover, in that case the components of the metric tensor are $C^{1 / 2}$-smooth functions of the distance coordinates, $[\mathbf{3 4}],[\mathbf{9 5}]$.

Distance coordinates were introduced in [35] and used there to obtain the first synthetic characterization of Riemannian manifolds as metric spaces which are both CBA and CBB with locally extendable and non-overlapping geodesics.

\section{Different types of convergence}

In general, the CBA-condition does not survive the Hausdorff-Gromov convergence. The reason is that the size of $\mathrm{CAT}(\kappa)$-neighborhoods may become arbitrarily small. The standard example is this: the hyperboloids $X_{\varepsilon}=\left\{(x, y, z) \in \mathbb{R}^{3}: x^{2}+y^{2}-z^{2}=\varepsilon^{2}\right\}$ with the induced intrinsic metrics are $\mathrm{CBA}(0)$ and they Hausdorff-Gromov converge to the double cone $X_{0}=\left\{(x, y, z) \in \mathbb{R}^{3}: x^{2}+y^{2}-z^{2}=0\right\}$ as $\varepsilon \rightarrow 0$ which is not CBA. However, we easily have: 
Theorem 5.1. If $X_{n} \rightarrow X$ by Hausdorff-Gromov and $X_{n}$ are $\mathrm{CBA}(\kappa)$ with convexity radii uniformly separated from 0 , then $X$ is $\mathrm{CBA}(\kappa)$.

One can define the Hausdorff-Gromov convergence as follows. The distortion of a map $f: X \rightarrow Y$ between metric spaces is

$$
\operatorname{dis}(f)=\sup _{x, x^{\prime} \in X}\left|d_{Y}\left(f(x), f\left(x^{\prime}\right)\right)-d_{X}\left(x, x^{\prime}\right)\right| .
$$

Let $A$ be the class of all maps $X \rightarrow Y$. Putting $\delta(X, Y)=\inf _{f \in A} \operatorname{dis}(f)$, one defines

$$
|X Y|_{H G}=\max \{\delta(X, Y), \delta(Y, X)\} .
$$

The convergence with respect to this metric is equivalent to the HausdorffGromov convergence.

Replacing the class $A$ of all maps by the class of all homeomorphisms, we arrive at the uniform metric and the uniform convergence respectively.

Now, we formulate a useful sufficient condition for retaining $\mathrm{CBA}(\kappa)$ under uniform convergence. Recall that the dilatation of a mapping $f$ between metric spaces $X, Y$ is the (possibly infinite) number

$$
\operatorname{dil}(f)=\sup \frac{\left|f(x) f\left(x^{\prime}\right)\right|}{\left|x x^{\prime}\right|}
$$

where the supremum is taken over all distinct $x, x^{\prime} \in X$.

We say that a sequence of metrics $\left\{d_{k}\right\}$ on $X$ has no local blow-ups if for every $x \in X$ there are $n \in \mathbb{N}$ and $\varepsilon>0$ such that the restriction $\mathrm{id}_{n, n+k}^{\varepsilon}$ of the identity map $\operatorname{id}_{n, n+k}:\left(X, d_{n}\right) \rightarrow\left(X, d_{n+k}\right)$ on the ball $B_{\varepsilon}^{d_{n}}(x)$ has dilatation $\operatorname{dil}\left(\mathrm{id}_{n, n+k}^{\varepsilon}\right) \leq C_{n}<\infty$ for all $k \in \mathbb{N}$. The following sufficient condition is proven in the lecture notes [53].

TheOREM 5.2. Assume that a sequence $\left\{d_{n}\right\}$ of metrics without local blow-ups on $X$ uniformly converges to a metric $d$. If $d_{n}$ is complete, $\mathrm{CBA}(\kappa)$ and its metric topology is locally compact for every $n \geq 1$, then $d$ is $\mathrm{CBA}(\kappa)$.

The condition that $d$ is a metric is essential as the example $X_{\varepsilon} \rightarrow X_{0}$ from above shows. Here we have the uniform convergence without blow-ups, however, the limiting $d$ is only a pseudo-metric.

Finally, we introduce the homotopy metric and the homotopy convergence which is well adapted to the CBA(0)-condition. Let $f: X \rightarrow Y$ be a homotopy equivalence with homotopy inverse $g: Y \rightarrow X$. For compact metric spaces $X, Y$ we put

$$
|X Y|_{h}=\inf _{f, g} \max \{\operatorname{dis}(f), \operatorname{dis}(g)\},
$$

where the infimum is taken over all homotopy equivalences $(f, g): X \leftrightarrow Y$. This homotopy distance defines a metric on the classes of isometric compact metric spaces. 
TheOREM 5.3. Assume that $\left|X_{n} X\right|_{h} \rightarrow 0$ as $n \rightarrow \infty$, where all $X_{n}$ are $\mathrm{CBA}(\kappa)$ with $\kappa \leq 0$. Then $X$ is $\mathrm{CBA}(\kappa)$.

This is proven in [19]. For $\kappa>0$, the $\mathrm{CBA}(\kappa)$-condition does in general not survive as the following example due to S. Ivanov [61] shows.

EXAMPLE 5.4. For every $\varepsilon>0$ there exists a contractible closed 2-polyhedron $X$ with a $\mathrm{CBA}(1)$-metric having diameter less than $\varepsilon$.

This polyhedron $X$ consists of a huge number of blocks $X_{i}$ (depending on $\varepsilon$ ), each of which is obtained as follows. Fix a tiny $\delta>0$ (depending on $\varepsilon$ ) and take a unit sphere $S^{2}$ with an open ball $B$ removed, where the boundary curve $\sigma$ of $B$ has length $\delta$. Next, take a tree $T \subset S^{2} \backslash B$ with the root vertex on $\sigma$ such that the length of every edge of $T$ is $\delta$ and whose vertices form a sufficiently dense subset in $S^{2}$. Finally, identify all vertices of $T$ getting $X_{i}$. Different blocks are glued together in a way such that the vertices $v_{i} \in X_{i}$ are identified with a unique vertex $v \in X$ and the boundary curve $\sigma_{i}$ of every block $X_{i}$ is identified with a curve of another block $X_{j}$ which originates from an edge of $T_{j}$. This gives the contractibility of $X$ (a similar effect provides the contractibility of the dunce cap). To ensure the CBA(1)-condition one should solve a combinatorial problem to guarantee the $2 \pi$-systole condition for the link of the vertex $v \in X$.

The question if it is possible to find similar $\varepsilon$-small CBA(1)-metrics on a fixed closed contractible 2-polyhedron remains open.

A volume convergence theorem for CBA-spaces is proven in [88] under the following assumptions.

Let $X, X_{j}, j \in \mathbb{N}$, be compact, geodesically complete $\mathrm{CBA}(\kappa)$-spaces of the same Hausdorff dimension $n$. Assume that the convexity radius $r\left(X_{j}\right)$ is separated from 0 uniformly in $j \in \mathbb{N}$ and $\left|X X_{j}\right|_{H G} \rightarrow 0$ as $j \rightarrow \infty$. Then $\mathcal{H}^{n}\left(X_{j}\right) \rightarrow \mathcal{H}^{n}(X)$.

\section{Constructions}

6.1. Gluing theorems. A simple but very useful tool is the following gluing theorem by Reshetnyak [98].

TheOREM 6.1. Let $X_{1}, X_{2}$ be complete locally compact $\mathrm{CBA}(\kappa)$-spaces. Suppose that there are convex sets $C_{i} \subset X_{i}$ and an isometry $f: C_{1} \rightarrow C_{2}$. Attach these spaces together along $f$. Then the resulting space $X$ is $\operatorname{CBA}(\kappa)$.

The proof is more or less straightforward and uses the comparison of angles. However, there is a standard trap while checking the condition of the theorem. $\mathbb{R}^{2}$ with an open disk removed is $\mathrm{CBA}(0)$ with convex boundary $C=\partial X$. Gluing two copies of $X$ along the boundary, we obtain a $\mathrm{CBA}(0)$ space $Y^{2}$. However, taking $\mathbb{R}^{n}$ with $n \geq 3$ instead of $\mathbb{R}^{2}$ we obtain a $Y^{n}$ which is not $\operatorname{CBA}(0)$. The reason is that $\mathbb{R}^{n}$ with an open ball removed is only $\operatorname{CBA}(\kappa)$ where $\kappa=r^{-2}$ and $r$ is the radius of the ball. Thus $Y^{n}$ is 
only $\mathrm{CBA}\left(r^{-2}\right)$. Similarly, the hyperbolic space $\mathrm{H}^{n}$ with an open horoball removed is only $\operatorname{CAT}(0)$ for $n \geq 3$, but not $\operatorname{CAT}(-1)$.

On the other hand, if we glue two copies of $\mathbb{R}^{n}$ along closed isometric balls, then the resulting space $Z^{n}$ is $\mathrm{CAT}(0)$ for every $n \geq 1$. These additional pieces which save the $\mathrm{CAT}(0)$-property are called fins and they were successfully used in the proofs of quite different results (see the end of sect. 6.2).

Trying to generalize Reshetnyak's gluing theorem, one can ask for conditions which would guarantee that gluing two smooth Riemannian manifolds $M_{1}, M_{2}$ of the same dimension $n \geq 3$ (the case $n=2$ we discuss in sect. 10) along isometric boundaries gives a $\mathrm{CBA}(\kappa)$-space, if both $M_{1}$, $M_{2}$ are $\mathrm{CBA}(\kappa)$. It is natural to conjecture that such a condition must be $L_{1}+L_{2} \leq 0$, where $L_{i}$ is the second fundamental form of $\partial M_{i}$. This was proven by N. Kosovskii, [68].

THEOREM 6.2. Assume that $L:=L_{1}+L_{2} \leq 0$ at the corresponding points of $\partial M_{1}, \partial M_{2}$. Then $M=M_{1} \cup M_{2}$ is locally a CBA-space. Moreover, if in addition the sectional curvatures of both $M_{1}, M_{2}$ are $\leq \kappa$ and the sectional curvatures of their common boundary $\Gamma$ are at most $\kappa$ at those 2 -directions where both $L_{1}, L_{2}$ are negatively determined, then $M$ is $\operatorname{CBA}(\kappa)$.

The example of the space $Y^{n}$ above shows that no condition can be omitted, and in fact the conditions above are necessary. Let $\Gamma \subset M$ be the singular hypersurface obtained from $\partial M_{1}, \partial M_{2}$ while gluing $M$. The approach is to extend the Riemannian metric appropriately, say of $M_{1} \subset M$ to a neighborhood of $\Gamma$ in $M$ and then using $L$ to perturb the metric on $M_{1}$ smoothing $\Gamma$ and pumping its singularity into the curvature of the perturbed metric. The main issue is to obtain a uniform (in the perturbation parameter $\delta$ ) curvature estimate from above. This is achieved via tremendous analytic calculations. The perturbed metrics form a sequence converging without local blow-ups to the initial one, which shows that the gluing gives a CBAspace.

Now, the sharp $\kappa$-estimate is obtained in two steps. First, it is obtained under the assumption $L<0$ using approaches from [14] and the fact that $M$ is CBA. Second, in the general case $L \leq 0$, the manifold $M$ is appropriately approximated by those with $L<0$. This is achieved by $C^{2}$-small changes of the metrics on $M_{1}, M_{2}$ in a way that the forms $L_{1}, L_{2}$ decrease while the induced metric on $\Gamma$ is not changed.

A sharp gluing CBB-theorem for two Riemannian manifolds is obtained by similar arguments in [69].

The result above is generalized in $[\mathbf{7 0}]$ to the case of an arbitrary finite number of manifolds $M_{a}, a \in A$, of the same dimension $n \geq 3$ glued together along the common boundary $\Gamma, M=\cup_{a} M_{a}$.

THEOREM 6.3. Assume that the sectional curvatures of the manifolds $M_{a}, a \in A$, are bounded from above by $\kappa$ and that $L_{a}+L_{a^{\prime}} \leq 0$ for each pair 
of different $a, a^{\prime} \in A$ and for the second fundamental forms $L_{a}, L_{a^{\prime}}$ of $\Gamma$ with respect to $M_{a}, M_{a^{\prime}}$ respectively. Furthermore, assume that the sectional curvatures of $\Gamma$ are bounded from above by $\kappa$ in those 2-directions where the forms $L_{a}, a \in A$, are negative definite simultaneously. Then $M$ is $\operatorname{CBA}(\kappa)$.

Again, the issue is to prove that $M$ is locally CBA. Then the sharp $\kappa$ estimate is obtained using methods of [14], see Proposition 3.6. Note that the condition is weaker than that by $[\mathbf{6 8}]$ yielding the $\mathrm{CBA}(\kappa)$ property for each union $M_{a} \cup M_{a^{\prime}}$ with different $a, a^{\prime} \in A$. It is only known from [68] that every such union is $\mathrm{CBA}\left(\kappa^{\prime}\right)$ for some $\kappa^{\prime} \in \mathbb{R}$.

To prove that $M$ is locally CBA, one needs a good control over minimizers in $M$. This is achieved under the assumption that the sums $L_{a}+L_{a^{\prime}}$ are locally uniformly negative by introducing a class of curves called almostgeodesics and careful study their properties (the general case $L_{a}+L_{a^{\prime}} \leq 0$ is obtained by approximation as in [68]). An almost-geodesic $\gamma$ in $M$ is a $C^{1}$ smooth curve concatenated from finitely many minimizers each of which is running in its own leaf $M_{a}$. Using a sort of linearization argument, the author shows that locally every almost-geodesic consists of at most three such minimizers, and the angle comparison with $M_{\kappa^{\prime}}$ holds for triangles formed by almost-geodesics. This suffices to prove that locally every minimizer in $M$ is almost-geodesic and therefore $M$ is locally CBA.

6.2. Warped products. Suppose that $B$ and $F$ are intrinsic metric spaces, and $f: B \rightarrow \mathbb{R}_{\geq 0}$ is continuous. Distance in the warped product $B \times_{f} F$ is defined by the infimum of path-lengths, where the length of a curve $\gamma=\left(\gamma_{B}, \gamma_{F}\right)$ for rectifiable curves $\gamma_{B}$ and $\gamma_{F}$ in $B$ and $F$ is given by:

$$
L(\gamma)=\int \sqrt{v_{B}^{2}(t)+f^{2}\left(\gamma_{B}(t)\right) v_{F}^{2}(t)} d t,
$$

where $v_{B}$ and $v_{F}$ are the speeds of $\gamma_{B}$ and $\gamma_{F}$. Equivalently, $L(\gamma)$ is the supremum of the expressions

$$
\sum\left(d_{B}\left(\gamma_{B}\left(t_{i}\right), \gamma_{B}\left(t_{i-1}\right)\right)^{2}+f^{2}\left(\gamma_{B}\left(t_{i}\right)\right) d_{F}\left(\gamma_{F}\left(t_{i}\right), \gamma_{F}\left(t_{i-1}\right)\right)^{2}\right)^{1 / 2} .
$$

For example, taking $F=S^{n-1}$, we obtain that

- for the function $f: B=[0, \infty) \rightarrow \mathbb{R}, f(t)=t$, the warped product space $B \times{ }_{f} F$ is isometric to $\mathbb{R}^{n}$ with the metric

$$
d s^{2}=d t^{2}+t^{2} d \omega_{n-1}^{2},
$$

where $d \omega_{n-1}^{2}$ is the standard metric of the unit sphere $S^{n-1}$;

- for the function $f: B=[0, \infty) \rightarrow \mathbb{R}, f(t)=\sinh t$, the warped product space $B \times_{f} F$ is isometric to $\mathrm{H}^{n}$ with the metric

$$
d s^{2}=d t^{2}+\sinh ^{2}(t) d \omega_{n-1}^{2} ;
$$


- for the function $f: B=[0, \pi] \rightarrow \mathbb{R}, f(t)=\sin t$, the warped product space $B \times{ }_{f} F$ is isometric to $S^{n}$ with the metric

$$
d s^{2}=d t^{2}+\sin ^{2}(t) d \omega_{n-1}^{2}
$$

The most general sharp conditions for a warped product of metric spaces to have a given curvature bound for CBA- as well as for CBB-spaces are found by S. Alexander and R. Bishop in [9]. We formulate these conditions for CBA-spaces.

Theorem 6.4. Let $B$ and $F$ be complete $\mathrm{CAT}(\kappa)$ and $\mathrm{CAT}\left(\kappa_{F}\right)$ spaces, respectively. Let $f: B \rightarrow \mathbb{R}_{\geq 0}$ be $\kappa$-convex, where $f$ is Lipschitz on bounded sets or $B$ is locally compact. Set $X=f^{-1}(0)$.

(1) If $X=\emptyset$, suppose $\kappa_{F} \leq \kappa(\inf f)^{2}$.

(2) If $X \neq \emptyset$, suppose $f^{\prime}\left(0^{+}\right)^{2} \geq \kappa_{F}$ at footpoints of $d_{X}$-minimizers in $B$, and $\kappa_{F} \leq \kappa f(p)^{2}$ for points $p \in B$ further than $\pi / 2 \sqrt{\kappa}$ from $X$.

Then $B \times{ }_{f} F$ is $\operatorname{CAT}(\kappa)$.

These conditions are close to be necessary ones. Namely, if a warped product of metric spaces $B \times{ }_{f} F$ has an upper curvature bound $\kappa$, then the same is true for $B$ because its images in $B \times{ }_{f} F$ are totally convex. One can also derive $\kappa$-convexity of the warped function $f$. It remains to show that $F$ has an upper curvature bound $\kappa_{F}$ satisfying conditions (1) and (2). This is obviously true if $f$ takes a positive minimum $f(p)$, since then $\{p\} \times F$ is totally convex.

As applications, the theorem above gives rise to a number of constructions of spaces with upper curvature bounds, among which is Reshetnyak's gluing theorem [98], which we discussed above (this theorem is used in the proof). It also covers a result of Ancel and Guilbault [16] saying that the interiors of compact contractible $n$-manifolds, $n \geq 5$, support a geodesic metric of strictly negative curvature.

The case of Hadamard spaces, that is, $\kappa=\kappa_{F}=0$ and $f>0$, was studied earlier in [7]. We briefly sketch the proof of that case underling the basic idea of the general proof. It can be illustrated as follows. Consider two copies of $\mathbb{R}^{n}, n \geq 1$, glued together along unit balls. Let $X_{n}$ be the resulting space, $Y_{n}=X_{n} \backslash$ (interior of $B$ ), where $B$ is the image of the balls. Then $X_{n}$ is $\mathrm{CBA}(0)$ (and even $\mathrm{CAT}(0)$ ) for every $n \geq 1$ by Reshetnyak's gluing theorem, while for $n \geq 3$ the space $Y_{n}$ is only $\mathrm{CBA}(1)$ and not $\mathrm{CBA}(0)$. This is because the boundary sphere of $B$ is convex in $Y_{n}$, and for $n \geq 3$ its dimension is bigger than 1. The effect of lowering a curvature bound by adding $B$ to $Y_{n}$ is crucial for the proof of the warped product theorem.

The proof proceeds by reduction to the case $F=\mathbb{R}$ and by approximation of $B \times{ }_{f} \mathbb{R}$ by subspaces $W_{\varepsilon}$ of Hadamard spaces $W_{\varepsilon}^{*}$ which are constructed as follows. We decompose the Euclidean product $B \times \mathbb{R}$ into three regions $W_{\varepsilon}^{(0)}=\{(p, u):-\varepsilon f(p) \leq u \leq \varepsilon f(p)\}, U_{\varepsilon}^{(0)}=\{(p, u): \varepsilon f(p) \leq u\}$, 
$L_{\varepsilon}^{(0)}=\{(p, u): u \leq-\varepsilon f(p)\}$, where the two last are closed convex subset because $f$ is convex. Taking isometric copies $W_{\varepsilon}^{(n)}, U_{\varepsilon}^{(n)}, L_{\varepsilon}^{(n)}$ of these, we construct $W_{\varepsilon}^{*}$ identifying isometric pairs $U_{\varepsilon}^{(n)}$ with $L_{\varepsilon}^{(n+1)}$ for each $n \in \mathbb{Z}$. To summarize, the space $W_{\varepsilon}^{*}$ consists of the mutually isometric strips $W_{\varepsilon}^{(n)}$ and the mutually isometric fins $U_{\varepsilon}^{(n)}=L_{\varepsilon}^{(n+1)}$ with the appropriate boundary components identified. Although the space $W_{\varepsilon}$ obtained by gluing together the strips $W_{\varepsilon}^{(n)}$ typically has positive infinite curvature, we recover nonpositive curvature by gluing on the fins.

The same sort of construction which takes into account fins has been used in $[\mathbf{5 0}]$ to prove a uniform estimate on the number of collisions in semi-dispersing billiards. The idea is to develop a billiard trajectory into a geodesic in a CBA-space obtained by gluing together step by step convex walls that are hit by the trajectory. This translates a difficult dynamical problem into a geometric one, which can be solved by geometric methods.

\section{Gauss equation}

The well known Gauss equation in Riemannian geometry allows to express intrinsic sectional curvatures of a submanifold via extrinsic curvatures and sectional curvatures of the ambient space. Surprisingly, the equation can be extended in a sense to arbitrary CBA-spaces. To describe such an extension, we recall some definitions.

Let $M$ be a $\operatorname{CBA}(\kappa)$-space, $\kappa \in \mathbb{R}$. A subset $N \subset M$ is said to have positive reach $\geq r$ if every point $x$ in the $r$-neighborhood of $N$ has a unique foot point in $N$, that is, $p \in N$ with $|x p|=\operatorname{dist}(x, N)$. It is proved in [77] that in the case $M$ is a Riemannian manifold, any subset $N \subset M$ of positive reach has some intrinsic curvature bound from above. The condition of positive reach can be expressed by comparing lengths of arcs and chords as follows [79]: a complete subset $N \subset M$ has positive reach if there exists $\rho>0$ such that intrinsic distances $d_{N}=s$ and extrinsic distances $d_{M}=r$ satisfy $s-r \leq C r^{3}$ for $r<\rho$ (actually, these two conditions are more or less equivalent for subsets of Riemannian manifolds). This estimate is an important step toward the notion of extrinsic curvature. The constant $C$ in front of $r^{3}$ on the right hand side may serve as a bound for extrinsic curvature. Namely, we say $N$ is a subspace of the extrinsic curvature $\leq A$ in $M$ if there is a length-preserving map $N \rightarrow M$ between intrinsic metric spaces, where $N$ is complete and

$$
s-r \leq \frac{A^{2}}{24} s^{3}+o\left(s^{3}\right)
$$

for all pairs of points having $s$ sufficiently small, [11]. For Riemannian submanifolds, this is equivalent to a bound, $|I I| \leq A$, on the second fundamental form. It is shown in [77] that subsets of bounded extrinsic curvature in a CBA-space are CBA-spaces with respect to their intrinsic metric. 
It follows from [6] that points of $N$ have neighborhoods in which $r$ is at least the chordlength of an arc of constant curvature $A$ and length $s$ in the model plane $M_{\kappa}$. The following sharp bound for subspaces of extrinsic curvature $\leq A$ is obtained in $[\mathbf{1 1}]$.

TheOrem 7.1 (Gauss equation). Suppose $N$ is a subspace of extrinsic curvature $\leq A$ in a $\mathrm{CBA}(\kappa)$-space. Then $N$ is $\operatorname{CBA}\left(\kappa+A^{2}\right)$.

This bound is realized by hypersurfaces of constant curvature in Euclidean, hyperbolic and spherical spaces.

The proof uses the knowledge that $N$ is $\operatorname{CBA}(K)$ for some $K$ by $[77]$, and RMT as a tool. However, the sharp bound requires rather involved and subtle arguments.

As an application, the following sharp estimate for the injectivity radius of a subspace is obtained in [11], which is new even in the case of Riemannian manifolds.

TheOREM 7.2. Suppose $N$ is a subspace of extrinsic curvature $\leq A$ in a $\mathrm{CAT}(\kappa)$ space. Then

$$
i n j_{N} \geq \min \left\{\frac{\pi}{\sqrt{\kappa+A^{2}}}, \frac{1}{2} c(A, \kappa)\right\},
$$

where $c(A, \kappa)$ is the circumference of a circle of curvature $A$ in $M_{\kappa}$.

\section{Extension results}

8.1. Lipschitz extension property. We say that a metric space $Y$ has the Lipschitz extension property (L) if there exists a constant $c \geq 1$ such that every $\lambda$-Lipschitz map $f: S \rightarrow Y$ defined on an arbitrary subset $S$ of some metric space $X$ can be extended to a $c \lambda$-Lipschitz map $\bar{f}: X \rightarrow Y$.

Obviously, to have property (L) is a bilipschitz invariant of $Y$. One can prove that the Lipschitz extension property implies that $Y$ is contractible.

A classical result of McShane [85] states that $\mathbb{R}$ has the property (L) with constant $c(\mathbb{R})=1$. The same result stays true for a metric tree. Applying this result to the coordinate functions, $\mathbb{R}^{n}$ has property $(\mathrm{L})$ with constant $c\left(\mathbb{R}^{n}\right)=\sqrt{n}$. Lang $[\mathbf{7 2}]$ showed that the optimal constant for $\mathbb{R}^{n}$ has to depend on $n$ and that $(\mathrm{L})$ is not valid for an infinite-dimensional Hilbert space.

In $[75]$ it is proven that

THEOREM 8.1. The following three classes of Hadamard spaces have the property $(L)$

(1) the 2-dimensional Hadamard manifolds;

(2) the class of Gromov-hyperbolic Hadamard manifolds whose curvature is bounded by $-b^{2} \leq K \leq 0$; 
(3) the class of homogeneous Hadamard manifolds and Euclidean Tits buildings.

The idea of the proof is as follows. Consider first an arbitrary Hadamard space $Y$ and a $\lambda$-Lipschitz map $f: S \rightarrow Y$ defined on a subset of a metric space $X$. In a first step, one associate to each $x \in X$ a bounded, closed convex set $A(x) \subset Y . A(x)$ is an intersection of closed balls centered at the points of $f(S)$ defined in the following way: We fix a constant $\alpha \geq 0$ and associate to each $x \in X$ the closed convex set

$$
A(x):=\bigcap_{s \in S} \bar{B}(f(s), \alpha \lambda d(x, s)) \subset Y,
$$

where $\bar{B}(y, r)$ is the closed ball of radius $r$ around $y$. Note that if $x \in S$, then $A(x)=\{f(x)\}$ since $f(x) \in \bar{B}(f(s), \lambda d(x, s))$ for all $s \in S$. One can prove the following:

(a) If $\alpha \geq \sqrt{2}$, then $A(x) \neq \emptyset$ for all $x \in X$.

(b) For $x, x^{\prime} \in X$ the Hausdorff distance between $A(x)$ and $A\left(x^{\prime}\right)$ satisfies

$$
\operatorname{Hd}\left(A(x), A\left(x^{\prime}\right)\right) \leq 2 \sqrt{2} \lambda d\left(x, x^{\prime}\right) .
$$

Hence, in order to extend $f$ to $X$, it would suffice to find a Lipschitz map $\phi: \mathcal{C} \rightarrow Y$ defined on the space $\mathcal{C}$ of bounded, closed convex subsets of $Y$, endowed with the Hausdorff distance, such that $\phi(\{y\})=y$ for all $y \in Y$.

It is not known and an interesting open question, if such a Lipschitz map $\phi$ exists for every finite dimensional Hadamard space (it is shown in [72] that the map $\phi$ does not exist for the Hilbert space $\ell_{2}$ ). However one can construct such a map in the cases (1), (2), (3) above.

In the extension results above we have only assumptions on the target space $Y$. If one also imposes assumptions on the source $X$, one can obtain better extension properties.

The following classical result is due to Kirszbraun, [64]: Let $X=Y=$ $\mathbb{R}^{n}, S \subset X$, then every 1-Lipschitz map $f: S \rightarrow Y$ has a 1-Lipschitz extension $\bar{f}: X \rightarrow Y$ of $f$.

There is a generalization of this result to the class of spaces, where the target space is $\operatorname{CAT}(\kappa)$ and the source space is $\operatorname{CBB}(\kappa)$ for the same $\kappa \in \mathbb{R},[\mathbf{7 3}]$.

Theorem 8.2. Let $\kappa \in \mathbb{R}$, and let $X, Y$ be two geodesic metric spaces such that $X$ is $\mathrm{CBB}(\kappa)$ and $Y$ is $\mathrm{CAT}(\kappa)$ and complete. Let $S$ be an arbitrary subset of $X$ and $f: S \rightarrow Y$ a 1-Lipschitz map with $\operatorname{diam} f(S) \leq D_{\kappa} / 2$. Then there exists a 1-Lipschitz extension $\bar{f}: X \rightarrow Y$ of $f$.

It suffices to extend $f$ to one additional point $x \in X \backslash S$. The general case follows inductively. One first looks for an optimal candidate $y \in Y$ as an image point of $x$. Therefore choose $\alpha \geq 0$ minimal such that the above defined set $A(x)=A_{\alpha}(x) \neq \emptyset$. In this case $A(x)=\{y\}$ consists of a single 
point $y$ which is the desired candidate. Comparing the space of directions in $x$ and $y$ using the scalar product of section 3.2 one can show that $\bar{f}(x)=y$ is actually a 1-Lipschitz extension.

8.2. Characterization of isometries. Let $X$ be a metric space, a bijective map $f: X \rightarrow X$ is an isometry, if it preserves all distances, i.e. for all $r \in(0, \infty)$ the following holds: if $x, y \in X$ then $d(x, y)=r$ if and only if $d(f(x), f(y))=r$.

What can we say about a map with the property that there exists some $r>0$ such that we have $d(x, y)=r$ if and only if $d(f(x), f(y))=r$ ? Note that there are nontranslational bijective (and continuous) maps $f: \mathbb{R} \rightarrow \mathbb{R}$ such that $|f(x+1)-f(x)|=1$ for all $x \in R$. Thus $f$ preserves the set of pairs of points with distance 1 . On the other hand, it is known from [27] that for $X=\mathbb{R}^{n}$ with $n \geq 2$, the bijection $f$ is an isometry. In the sixties, A.D. Alexandrov posed the problem to describe the class of metric spaces $X$, for which all bijections $X \rightarrow X$ preserving distance 1 are isometries. There is a number of results in this direction. We mention only that the hyperbolic spaces $\mathrm{H}^{n}, n \geq 2$, are in that class according to [71]. Moreover, it turned out that a large subclass of $\operatorname{CAT}(\kappa)$-space with $\kappa \leq 0$ is also there. More precisely, the following is proved in $[\mathbf{3 2}]$.

THEOREM 8.3. Let $\kappa<0$ and let $X$ be a locally compact, geodesically complete $\mathrm{CAT}(\kappa)$-space whose boundary at infinity is connected. Let $f: X \rightarrow$ $X$ be a bijective map such that there exists $r>0$ such that $d(x, y)=r$ if and only if $d(f(x), f(y))=r$. Then $f$ is an isometry.

P. Andreev generalized this result to the case of $\mathrm{CAT}(0)$ spaces which are Busemann G-spaces in [17], and finally to general CAT $(0)$ spaces in [18], that is, Theorem 8.3 holds true also for $\kappa=0$.

\section{Rigidity results}

Let $X$ be a Hadamard manifold. For every point $x \in X$, we have an involutive homeomorphism $\phi_{x}: \partial_{\infty} X \rightarrow \partial_{\infty} X$ of the boundary at infinity defined as follows. Given $\xi \in \partial_{\infty} X$, there is a unique geodesic ray $\gamma:[0, \infty) \rightarrow X$ with $\gamma(0)=x$ asymptotic to $\xi, \gamma(\infty)=\xi$. Then we put $\phi_{x}(\xi)=\gamma^{\prime}(\infty)$, where the ray $\gamma^{\prime}, \gamma^{\prime}(0)=x$, is opposite to $\gamma, \frac{d \gamma^{\prime}}{d t}(0)=-\frac{d \gamma}{d t}(0)$. Clearly, $d_{T}(\xi, \phi(\xi)) \geq \pi$ for the Tits metric $d_{T}$ on $\partial_{\infty} X$.

A subset $A \subset \partial_{\infty} X$ is said to be involutive if it is invariant under all involutions $\phi_{x}, x \in X$.

For example, if $X=X_{1} \times X_{2}$ is the metric product, then both subsets $\partial_{\infty} X_{i} \subset \partial_{\infty} X, i=1,2$, are proper, involutive and closed. In this example, the Tits boundary $\partial_{T} X$ is a spherical join, $\partial_{T} X=\partial_{T} X_{1} * \partial_{T} X_{2}$.

Another important example is the set of singular points $S \subset \partial_{\infty} X$ of a higher rank symmetric space $X$ of noncompact type, that is, $\xi \in S$ if and only if $\xi$ is the endpoint at infinity of a singular geodesic ray $\gamma \subset X$. 
Again, the subset $S$ is proper, involutive and closed. In this example, the Tits boundary $\partial_{T} X$ is a spherical building.

The famous higher rank rigidity $[\mathbf{2 2}, \mathbf{5 2}]$ can be established using the following result of P. Eberlein (for detailed exposition see [56]):

Assume that the boundary at infinity $\partial_{\infty} X$ of a Hadamard manifold $X$ contains a proper, involutive, closed (in the cone topology) subset $A$. Then the holonomy group of $X$ is not transitive.

Combined with well a known characterization of products and symmetric spaces $[\mathbf{4 1}, \mathbf{1 0 3}]$, this yields:

Under the condition above, $X$ is a product or a symmetric space and therefore $\partial_{T} X$ is a spherical join or a building.

That is the context in which the following result [80] should be considered. Recall that the Tits boundary $\partial_{T} X$ of each Hadamard space $X$ is $\operatorname{CAT}(1)$.

THEOREm 9.1. Let $X$ be a finite dimensional geodesically complete CAT (1) space. If $X$ has a proper closed subset $A$ containing with each $a \in A$ all antipodes of $a$, i.e. all points $x \in X$ with $|x a| \geq \pi$, then $X$ is a spherical join or building.

This result is also related to the characterization [76] of affine buildings or symmetric spaces as those geodesically complete locally compact Hadamard spaces $X$ that have a non-discrete irreducible spherical building as the Tits boundary $\partial_{T} X$.

As a consequence, we have a rigidity property of spherical buildings and joins.

Corollary 9.2. Let $X$ be a non-discrete spherical building or a spherical join. If $f: X \rightarrow Y$ is a surjective 1-Lipschitz map onto a finitedimensional geodesically complete CAT(1) space, then $Y$ is also a spherical building or a spherical join too.

Typical examples of surjective 1-Lipschitz maps as above arise as follows. Let $X$ be a Hadamard space and let $\Sigma_{x}$ be the space of directions of $X$ at some point $x \in X$. The map $f: \partial_{T} X \rightarrow \Sigma_{x}$ that assigns to every $\xi \in \partial_{T} X$ the direction $f(\xi) \in \Sigma_{x}$ of the unique geodesic ray $x \xi \subset X$ is surjective and 1-Lipschitz. There are four basic cases:

- $X$ is a product. Then $\partial_{T} X, \Sigma_{x}$ are spherical joins.

- $X$ is hyperbolic. Then $\partial_{T} X$ is discrete.

- $X$ is an affine building. Then $\partial_{T} X, \Sigma_{x}$ are spherical buildings of one and the same dimension, and $f$ folds a lot.

- $X$ is an irreducible symmetric space of higher rank $k \geq 2$. Then $\partial_{T} X$ is a spherical building of dimension $k-1, \Sigma_{x}$ is the unit sphere $S^{m-1}$, where $m=\operatorname{dim} X$ might be much larger than $k$. 
A subset $A \subset X$ of a $\mathrm{CAT}(1)$ space $X$ is said to be symmetric if it contains all antipodes of its points, $\operatorname{ant}(A) \subset A$. The following simple observation is at the very beginning of the rigidity above.

Let $A$ be a subset in a geodesically complete CAT(1) space $X$, and $x^{\prime} \in X$ an antipode of $x \in X$. Then $\operatorname{dist}\left(x^{\prime}, \operatorname{ant}(A)\right) \leq \operatorname{dist}(x, A)$. In the case of equality, we have $\left|x x^{\prime}\right|=\pi$, and for every $a \in A$ with $\operatorname{dist}(x, A)=|x a|$, the equality $|x a|+\left|a x^{\prime}\right|=\pi$ holds, i.e., $x a x^{\prime}$ is a geodesic.

For the proof, we can assume that $\left|a x^{\prime}\right|<\pi$ for a given $a \in A$. Extending the geodesic segment $a x^{\prime}$ at the right end up to the length $\pi$, we find $a^{\prime} \in$ $\operatorname{ant}(A)$ with $x^{\prime} \in a a^{\prime},\left|a a^{\prime}\right|=\pi$. Then we have $\left|a x^{\prime}\right|+\left|x^{\prime} a^{\prime}\right|=\pi \leq\left|x x^{\prime}\right| \leq$ $|x a|+\left|a x^{\prime}\right|$, therefore $\left|x^{\prime} a^{\prime}\right| \leq|x a|$. Hence, the claim.

Given $x \in X$, we let $A_{x} \subset X$ be the minimal symmetric subset that contains $x$. Then $y \in A_{x}$ is equivalent to $x \in A_{y}$, and therefore the sets $A_{x}, x \in X$, define a decomposition of $X$ into minimal symmetric subsets. As a consequence of the lemma above, we see that this decomposition is equidistant, that is, $\operatorname{dist}\left(A_{x}, y\right)=\operatorname{dist}\left(A_{x}, A_{y}\right)=\operatorname{dist}\left(x, A_{y}\right)$ for each $x, y \in$ $X$. Therefore, the quotient map $\delta: X \rightarrow \Delta_{X}=X /\left\{A_{x}\right\}$ is a submetry, that is, for each $x \in X$ and $r>0$, the map sends the (closed) $r$-ball around $x$ onto the (closed) $r$-ball around $\delta(x)$. The notion of a submetry was introduced by V. Berestovskii, see $[\mathbf{3 6}, \mathbf{3 7}, \mathbf{3 8}]$. For a comprehensive account of submetries see also $[\mathbf{8 1}]$. A careful study of the submetry $\delta$ and of some of its refinements leads to the proof of Theorem 9.1.

\section{2-dimensional polyhedra}

In the case of 2-polyhedra, we have at our disposal a more or less complete description of general CBA-metrics. Several new effects, which are absent for surfaces, arise for polyhedra with topological singularities.

10.1. Singular edges are curves with bounded turn variation. Let $Y$ be the union of three rays with the common vertex $v, X=Y \times \mathbb{R}$. Then $l=v \times \mathbb{R} \subset X$ is the (topologically) singular edge. The important fact is that for every CBA-metric on $X$, the edge $l$ has bounded turn variation. It was formulated as a question in $[\mathbf{2 6}]$ and then proven by B. Kleiner (unpublished).

The explanation (not the proof!) is very simple. Assume that we have a CBA-metric on $Y$, in which $l$ is piecewise geodesic. The link of every point $x \in l$ is the bipartite graph $L$ with two vertices and three edges between them. The CBA-condition implies that the systole of $L$ is at least $2 \pi$. Now, if $x$ is a (metric) vertex with a small angle from one of the faces, then the length of the corresponding edge of $L$ is short. Hence, the lengths of both other edges must be large to satisfy the systole condition. Thus we have a large negative curvature at $x$. This is a mechanism which translates turns of a singular edge into negative curvature of the polyhedron. If $l$ would have the unbounded turn variation, then it would imply a huge 
accumulation of negative curvature along $l$ which would destroy the topology of the polyhedron. This explains why singular edges are curves with the bounded turn variation $|\tau|$ w.r.t. a tame CBA-metric, which is the uniform limit of piecewise smooth CBA-metrics. An efficient estimate of $|\tau|$ follows from the Gauss-Bonnet formula.

\subsection{Signed curvature measure and the Gauss-Bonnet formula} for tame metrics. Let $X$ be a 2-polyhedron with a piecewise smooth metric $d$. For a face $f \subset X$, let $K$ denote the Gaussian curvature of $f$. The curvature of a Borel subset $B \subset f$ is defined by

$$
\omega(B)=\int_{B} K \mathrm{~d} \sigma
$$

where $\sigma$ is the area measure of $f$.

For an edge $e$ and a face $f$ adjacent to $e$, we denote by $\tau_{f}$ the turn of $e$ from the side of $f$, that is, for a Borel subset $B \subset e, \tau_{f}(B)=\int_{B} k_{f} \mathrm{~d} s, k_{f}$ being the geodesic curvature of $e$ with respect to $f$. The sign of $k_{f}$ is chosen in such a way that $k_{f}$ is positive for a convex $f$. By definition, the curvature of a Borel subset $B \subset e$ is the sum of the turns from the side of all faces adjacent to $e$,

$$
\omega(B)=\sum_{f \mid e} \tau_{f}(B) .
$$

For a vertex $v$, let $\chi(v)=\chi\left(\Lambda_{v}\right)$ be the Euler characteristic of the link of $v$ in $X$. On $\Lambda_{v}$, the metric $d$ induces the angle (pseudo-)metric $\alpha_{d}$ in which the length of an edge corresponding to a face $f \subset X$ is the angle $\alpha(v, f)$ of this face at $v$. Let $\alpha(v)$ be the length of the link $\Lambda_{v}$ with respect to $\alpha_{d}$, i.e., the sum of the lengths of all edges of $\Lambda_{v}$. By definition, the curvature of $v$ is

$$
\omega(v)=(2-\chi(v)) \pi-\alpha(v) .
$$

By additivity, these definitions extend to the Borel subsets $B \subset X$. This defines the signed curvature measure $\omega$ of a piecewise smooth metric on $X$. Now, we have the Gauss-Bonnet formula

$$
\omega(X)=2 \pi \chi(X)
$$

for $d$, where $\chi(X)$ is the Euler characteristic of $X,[\mathbf{2 6}]$. The notion of the signed curvature measure $\omega$ and the Gauss-Bonnet formula have been extended to the tame CBA-metrics on $X$ in [19]. Using it, the following estimate of turn was obtained there.

TheOrem 10.1. Let $X$ be a closed 2-polyhedron. For the turn variation of the essential 1-skeleton of $X$ with respect to a $\mathrm{CBA}(\kappa)$-metric $d$ we have

$$
|\tau|\left(\operatorname{esk}_{1} X\right) \leq c_{1}+c_{2} \cdot \omega\left(X \backslash \operatorname{esk}_{1} X\right)
$$

where $c_{1}, c_{2}$ are constants depending only on the topology of $X$. In particular, $|\tau|\left(\operatorname{esk}_{1} X\right) \leq c_{1}+c_{2} \cdot \kappa \operatorname{Area}(X, d)$. 
10.3. Gluing condition, characterization and approximation theorems. We denote by $\mathcal{R}_{\kappa}$ the class of locally compact 2-polyhedra with a CBA $(\kappa)$-metric, all boundary edges of which are curves of finite turn variation. Let $M_{i}$ be a domain on a surface of class $\mathcal{R}_{\kappa}$ having the compact closure $\bar{M}_{i}$ and bounded by finitely many curves of finite turn variation (some of these curves may degenerate to points). We glue a polyhedron $X$ from a collection of such domains $M_{i}$ requiring that the following two conditions are fulfilled.

(i) For any Borel subset $B$ of an arbitrary edge $e \subset X$ and any domains $M_{i}, M_{j}$ adjacent to $e$, we have

$$
\tau_{i}(B)+\tau_{j}(B) \leq 0,
$$

where $\tau_{i}, \tau_{j}$ are the turns of $e$ from the (different!) sides of $M_{i}, M_{j}$ respectively.

(ii) For any vertex $x \in X$, the length of each noncontractible loop in the link $\Lambda_{x}$ is at least $2 \pi$.

For CBA-metrics on 2-polyhedrons we have a generalisation of Reshetnyak's gluing theorem.

Theorem 10.2. A polyhedron $X$ glued together from surfaces $M_{i} \in \mathcal{R}_{\kappa}$ is of class $\mathcal{R}_{\kappa}$ if and only if the conditions (i), (ii) are fulfilled.

The proof is based on the following Limit Metric Theorem.

THEOREM 10.3. Assume that a metric $d$ on a compact 2-polyhedron $X$ is the uniform limit of a sequence $d_{n} \in \mathcal{R}_{\kappa}$ such that the positive curvature parts $\omega_{n}^{+}$of these metrics are uniformly bounded on $X \backslash \operatorname{esk}_{1} X$ and the turn variations of the boundary edges are uniformly bounded. Then $d \in \mathcal{R}_{\kappa}$.

This was proven in [49] under the additional condition that the lengths of esk ${ }_{1} X$ are uniformly bounded. In [60], it was shown that this condition follows from the others. The main issue in the proof of the Limit Metric Theorem is to obtain a uniform separation from zero of the convexity radius for metrics $d_{n}$.

Furthermore, we have a Characterization Theorem of CBA-metrics on 2-polyhedra:

THEOREM 10.4. Each polyhedron $X \in \mathcal{R}_{\kappa}$ can be glued together from surfaces $M_{i} \in \mathcal{R}_{\kappa}$ in such a way that the conditions (i), (ii) are fulfilled.

The proof is based on the following Approximation Theorem [49].

THEOREM 10.5. Every metric $d \in \mathcal{R}_{\kappa}$ on a locally compact 2-polyhedron $X$ is the uniform limit of a sequence of piecewise smooth metrics $d_{n} \in \mathcal{R}_{\kappa}$ on $X$ such that the curvature variations $\left|\omega_{n}\right|$ of $d_{n}$ are (locally) uniformly bounded on $X \backslash \operatorname{esk}_{1} X$, the essential 1-skeleton esk $_{1} X$ has (locally) uniformly bounded lengths, and the boundary edges have (locally) uniformly bounded turn variations. 
The proof essentially uses the infinitesimal theory of locally compact CBA-spaces due to B. Kleiner.

It would be very desirable to replace the condition in the Limit Metric Theorem that the metric $d$ is a certain uniform limit by the weaker condition that $d$ is the homotopy limit of metrics $d_{n}$ on the corresponding 2-polyhedra $X_{n}$. However, this is still an open question.

10.4. Rigidity due to Gauss-Bonnet. There is another remarkable geometric effect in the class of 2-polyhedra with a NPC-metric which is completely invisible for surfaces. To start with, we consider the following invariant of a finite graph $\Lambda$,

$$
\sigma(\Lambda):=\inf _{\alpha} \frac{\alpha(\Lambda)}{\operatorname{sys}(\alpha)}
$$

the size of $\Lambda$, where $\alpha$ is a length (pseudo)-metric on $\Lambda, \alpha(\Lambda)$ the length of $\Lambda$, sys $(\alpha)$ the length of a shortest essential loop in $\Lambda$. Next, we put

$$
\omega(\Lambda):=(2-\chi(\Lambda)) \pi-2 \pi \sigma(\Lambda),
$$

the maximal total curvature of $\Lambda$. Then $\omega_{\alpha}(\Lambda):=(2-\chi(\Lambda)) \pi-\alpha(\Lambda) \leq \omega(\Lambda)$ for every length metric $\alpha$ on $\Lambda$ with $\operatorname{sys}(\alpha) \geq 2 \pi$. A length metric $\alpha$ is called minimal if $\omega_{\alpha}(\Lambda)=\omega(\Lambda)$, which is equivalent to $\alpha(\Lambda)=2 \pi \sigma(\Lambda)$.

A NPC-metric $d$ on a closed 2-polyhedron $X$ is tight if all maximal surfaces of $X$ are flat, all maximal essential edges of $X$ are geodesics and for any essential vertex $v \in X$ the induced angle metric $\alpha_{d}$ on the link $\Lambda_{v}$ is minimal.

TheOREm 10.6. Assume that a closed 2-polyhedron $X$ admits a NPCmetric. Then $\sum_{v \in V_{e}} \omega\left(\Lambda_{v}\right) \geq 2 \pi \chi(X)$ and equality holds if and only if one, and hence any, NPC-metric of $X$ is tight.

This easily follows from Gauss-Bonnet. The reason is that for the summands of Gauss-Bonnet

$$
2 \pi \chi(X)=\sum_{v} \omega_{\alpha}\left(\Lambda_{v}\right)+\sum_{f} \omega(f)+\sum_{e} \omega(e)
$$

we have $\omega(f), \omega(e) \leq 0, \omega_{\alpha}\left(\Lambda_{v}\right) \leq \omega\left(\Lambda_{v}\right)$ for any NPC-metric on $X$, and these equalities imply that the metric is tight.

The examples of polyhedra with tight metrics include amongst others branched coverings of degree 2 over a 2-skeleton of $n$-simplex, $n \geq 4$ and factor spaces $Y / \Gamma$, where $Y$ is a thick Euclidean 2-building and $\Gamma$ is a properly discontinuous and cocompact group of automorphisms of $X$. Furthermore, there are closed 2-polyhedra with Gromov hyperbolic fundamental group carrying tight NPC-metrics. Such a polyhedron admits no CAT(-1)-metric, [26], and moreover, its fundamental group admits no discrete, cocompact action on any $\mathrm{CAT}(-1)$ 2-polyhedron, [62]. 
10.5. Metrics with bounded total curvature. For more than forty years, there exists a theory of metrics on surfaces which includes CBAand CBB-metrics as rather particular cases. These are the metrics with bounded total curvature (BTC-metrics) synthetically defined by the requirement that the total excess of any system of nonoverlapping geodesic triangles be (locally) uniformly bounded, $[\mathbf{3}]$. From an equivalent analytical point of view, such a metric can locally be given by

$$
d s^{2}=\lambda(x, y)\left(d x^{2}+d y^{2}\right),
$$

where $\ln \lambda(x, y)$ is the difference of two subharmonic functions, [99]. This class of metrics is closed in the topology of the uniform convergence, and curves with the bounded turn variation play a key role for a gluing theorem and the Gauss-Bonnet formula. Gluing two BTC-surfaces along boundaries which are curves with the bounded turn variation gives a BTC-surface.

All this together with the characterization theorem for CBA-metrics on 2-polyhedra allows to suggest that there should exist a theory of BTCmetrics on 2-polyhedra. However, it is even unclear how to define such a metric: any straightforward generalization of the surface case fails.

A particular related question is the following. Let $Y$ be again the union of three rays with the common vertex $v, X=Y \times \mathbb{R}^{2}$. Assume the a CBAmetric on $X$ is given. Is it true that the metric induced on the singular edge $v \times \mathbb{R}^{2}$ has bounded total curvature?

\section{References}

[1] A.D. Aleksandrov, A theorem on triangles in a metric space and some of its applications, (Russian) Trudy Mat. Inst. Steklov., v. 38, pp. 5-23. Trudy Mat. Inst. Steklov., v 38, Izdat. Akad. Nauk SSSR, Moscow, 1951.

[2] A. Aleksandrov, Über eine Verallgemeinerung der Riemannschen Geometie, Schr. Forschungsinst. Math. 1 (1957), 33-84.

[3] A. Aleksandrov, V. Zalgaller, Intrinsic geometry of surfaces, Transl. Math. Monogr., vol. 15, Amer. Math. Soc., Providence, RI, 1967.

[4] A.D. Aleksandrov, V.N. Berestovskii, I.G. Nikolaev, Generalized Riemannian spaces, Russian Math. Surveys 41 (1986), 1-54.

[5] S. Alexander, R. Bishop, The Hadamard-Cartan theorem in locally convex metric spaces, L'Enseig. Math., 36 (1990), 309-320.

[6] S. Alexander, R. Bishop, Comparison theorems for curves of bounded geodesic curvature in metric spaces of curvature bounded above, Differential Geom. Appl. 6 (1996), no. $1,67-86$.

[7] S. Alexander, R. Bishop, Warped products of Hadamard spaces, Manuscripta Math. 96 (1998), 487-505.

[8] S. Alexander, R. Bishop, FK-convex functions on metric spaces, Manuscripta Math. 110 (2003), no. 1, 115-133.

[9] S. Alexander, R. Bishop, Curvature bounds for warped products of metric spaces, Geom. Funct. Anal. 14 (2004), no. 6, 1143-1181.

[10] S. Alexander, R. Bishop, A cone splitting theorem for Alexandrov spaces, Pac. J. Math. 218 (2005), 1-16.

[11] S. Alexander, R. Bishop, Gauss equation and injectivity radii for subspaces in spaces of curvature bounded above, Geom. Dedicata 117 (2006), 65-84. math.DG/0511570. 
[12] S. Alexander, R. Bishop, Comparison theorems for curves of bounded geodesic curvature in metric spaces of curvature bounded above, Differential Geom. Appl. 6 (1996), no. $1,67-86$.

[13] S. Alexander, I. Berg, R. Bishop, The Riemannian obstacle problem, Illinois J. Math. 31 (1987), 167-184.

[14] S. Alexander, I. Berg, R. Bishop, Geometric curvature bounds in Riemannian manifolds with boundary, Trans. Amer. Math. Soc. 339 (1993), no. 2, 703-716.

[15] F. Ancel, M. Davis, C. Guilbault, CAT(0) reflection manifolds, Geometric topology (Athens, GA, 1993), 441-445, AMS/IP Stud. Adv. Math., 2.1, Amer. Math. Soc., Providence, RI, 1997.

[16] F. Ancel, C. Guilbault, Interiors of compact contractible n-manifolds are hyperbolic $(n \geq 5)$, J. Differ. Geom. 45 (1997), 1-32.

[17] P. Andreev, Recovering the metric of a CAT(0)-space by a diagonal tube, J. Math. Sci. (N. Y.) 131 (2005), 5257-5269; translated from Zap. Nauchn. Sem. POMI, 299 (2003), 5-29.

[18] P. Andreev, A.D. Aleksandrov's problem for CAT(0)-spaces, Siberian Math. J. 47 (2006), no. 1, 1-17.

[19] I. Arshinova, S. Buyalo, Metrics of upper bounded curvature on 2-polyhedra, St. Petersburg Math. J., 8 (1997), 825-844.

[20] W. Ballmann, Singular spaces of non-positive curvature, Chapter 10 of Sur les Groupes Hyperboliques d'après Mikhael Gromov (ed. E. Ghys, P. de la Harpe), Progress in Maths. 83, Birkhäuser (1990).

[21] W. Ballmann, Lectures on spaces of nonpositive curvature, DMV Seminar 25, Birkhäuser Verlag, Basel [et al.], 1995.

[22] W. Ballmann, Nonpositively curved manifolds of higher rank, Ann. of Math. 122:3 (1985), 597-609.

[23] W. Ballmann, M. Brin, Orbihedra of nonpositive curvature, Publications Math. IHES 82 (1995), 169-209.

[24] W. Ballmann, M. Brin, Diameter rigidity of spherical polyhedra, Duke Math. J. 97 (1999), 235-259.

[25] W. Ballmann, M. Brin, Rank rigidity of Euclidean polyhedra, Amer. J. Math. 122 (2000), 873-885.

[26] W. Ballmann, S. Buyalo, Nonpositively curved metrics on 2-polyhedra, Math. Z. 222 (1996), 97-134.

[27] F. Beckman, D. Quarles, On isometries of Euclidean spaces, Proc. Amer. Math. Soc. 4 (1953), 810-815.

[28] W. Ballmann, M. Gromov, V. Schroeder, Manifolds of Nonpositive Curvature, Progress in Math. vol. 61, Birkhäuser, Boston, 1985.

[29] V. Berestovskii, Borsuk's problem on the metrization of a polyhedron, Soviet Math. Dokl. 27 (1983), 56-59.

[30] V. Berestovskii, On A.D. Aleksandrov spaces of curvature bounded above, Dokl. Akad. Nauk 342 (1995), 304-306.

[31] V. Berestovskii, Pathologies in Alexandrov spaces with curvature bounded above, Siberian Adv. Math. 12 (2002), no. 4, 1-18 (2003).

[32] V. Berestovskii, Isometries in Aleksandrov spaces of curvature bounded above, Illinois J. Math. 46 (2002), no. 2, 645-656.

[33] V. Berestovskii, Busemann spaces with upper bounded Aleksandrov curvature, St. Petersburg Math. J. 14 (2003), no. 5, 713-723.

[34] V. Berestovskii, Manifolds with an intrinsic metric with one-sided bounded curvature in the sense of A.D. Aleksandrov, Mat. Fiz. Anal. Geom. 1 (1994), no. 1, 41-59 (Russian).

[35] V. Berestovskii, Introduction of a Riemannian structure into certain metric spaces, Siberian Math. J. 16 (1975), no. 4, 499-507. 
[36] V. Berestovskii, "Submetries" of three-dimensional forms of nonnegative curvature, (Russian) Sibirsk. Mat. Zh. 28 (1987), no. 4, 44-56, 224.

[37] V. Berestovskii, A metric characterization of Riemannian submersions for A.D. Aleksandrov manifolds of bounded curvature, (Russian) Proceedings of the Conference "Geometry and Applications" dedicated to the seventieth birthday of V. A. Toponogov (Novosibirsk, 2000), 11-16, Ross. Akad. Nauk Sib. Otd., Inst. Mat., Novosibirsk, 2001.

[38] V. Berestovskii, L. Guijarro, A metric characterization of Riemannian submersions, Ann. Global Anal. Geom. 18 (2000), no. 6, 577-588.

[39] V. Berestovskii, I. Nikolaev, Multidimensional generalized Riemannian spaces, in Geometry IV. Non-regular Riemannian Geometry. Encyclopaedia of Math. Sciences Springer-Verlag, Berlin, Heidelberg, 165-244 (1993).

[40] I. Berg, I. Nikolaev, On a distance between directions in an Aleksandrov space of curvature $\leq K$, Michigan Math. J. 45 (1998), no. 2, 257-289.

[41] M. Berger, Sur les groupes d'holonomie des variétés riemanniennes, Bull. Soc. Math. France 83 (1955), 279-330.

[42] R. Bishop, Jordain domains are CAT(0), math.DG/0512622.

[43] M. Bourdon and H. Pajot, Poincaré inequalities and quasiconformal structure on the boundary of some hyperbolic buildings, Proc. of Amer. Math. Soc. 127 (1999), no. 8, 2315-2324.

[44] T. Brady, Complexes of nonpositive curvature for extensions of $F_{2}$ by $Z$, Topology Appl. 63 (1995), no. 3, 267-275.

[45] T. Brady, Complexes of non-positive curvature and automorphisms of the 4-punctured sphere, Arch. Math. (Basel) 67 (1996), no. 2, 173-176.

[46] M. Bridson, A. Haefliger, Metric spaces of non-positive curvature, Springer-Verlag, Berlin - Heidelberg, 1999.

[47] Yu. Burago, M. Gromov, G. Perelman, A.D. Alexandrov spaces with curvatures bounded from below, Uspekhi Mat. Nauk 47 (1992), no. 2, 3-51 (Russian); English transl., Russian Math. Surveys 47 (1992), no. 2, 1-58.

[48] D. Burago, Yu. Burago, S. Ivanov, A Course in Metric Geometry, Grad. Stud. Math., Amer. Math. Soc. 33, Providence, 2001.

[49] Yu. Burago, S. Buyalo, Metrics of upper bounded curvature on 2-polyhedra. II, St. Petersburg Math. J. 10 (1999), 619-650.

[50] D. Burago, S. Ferleger, A. Kononenko, Uniform estimates on the number of collisions in semi-dispersing billiards, Ann. of Math. 147 (1998), 695-708.

[51] M. Bonk, O. Schramm, Embeddings of Gromov hyperbolic spaces, Geom. Funct. Anal. 10 (2000), 266-306.

[52] K. Burns, R. Spatzier, Manifolds of nonpositive curvature and their buildings, Inst. Hautes Études Sci. Publ. Math. 65 (1987), 35-59.

[53] S. Buyalo, Spaces of curvature bounded above, S. Petersburg, Obrazovanie, 1997 (Russian)

[54] J. Cheeger, Differentiability of Lipschitz functions on metric measure spaces, Geom. and Funct. Anal. 9 (1999), 428-517.

[55] M. Davis, T. Januszkiewicz, Hyperbolization of polyhedra, J. Differ. Geom. 34 (1991), 347-388.

[56] P. Eberlein, Geometry of nonpositively curved manifolds, Chicago Lectures in Mathematics, 1996.

[57] K. Fujiwara, T. Shioya, S. Yamagata, Parabolic isometries of CAT(0) spaces and CAT(0) dimensions, Algebr. Geom. Topol. 4 (2004), 861-892 (electronic).

[58] M. Gromov, CAT $(\kappa)$-spaces: constructions and concentration, J. Math. Sci. (N. Y.) 119 (2004), no. 2, 178-200.

[59] M. Gromov, R. Schoen, Harmonic maps into singular spaces and p-adic superrigidity for lattices in groups of rank one, Publications Math. IHES, 76 (1992), 165-246. 
[60] S. Ivanov, On convergent metrics of upper-bounded curvature on 2-polyhedra, St. Petersburg Math. J. 10 (1999), no. 4, 663-670.

[61] S. Ivanov, A contractible geodesically complete space of curvature $\leq 1$ with arbitrarily small diameter, St. Petersburg Math. J. 13 (2002), no. 4, 593-599.

[62] M. Kapovich, An example of 2-dimensional hyperbolic group which can't act on 2-dimensional negatively curved complexes, Preprint 1994.

[63] H. Karcher, Riemannian comparison constructions, S.S Chern, (ed.), Global Differential Geometry, MAA Studies in Math., 27, Math. Assoc. Amer. 1987, 170-222.

[64] M. Kirszbraun, Über die zusammenziehende und Lipschitzsche Transformationen, Fundamenta Math. 22 (1934), 77-108.

[65] B. Kleiner, The local structure of length spaces with curvature bounded above, Math. Z. 231 (1999), 409-456.

[66] B. Kleiner, B. Leeb, Rigidity of quasi-isometries for symmetric spaces and Euclidean buildings, Inst. Hautes Études Sci. Publ. Math. 86 (1997), 115-197.

[67] N. Korevaar, R. Schoen, Sobolev spaces and harmonic maps for metric space targets, Communications in Analysis and Geometry, 1(3-4) (1993), 561-659.

[68] N. Kosovskiı̌, Gluing of Riemannian manifolds of curvature $\leq \kappa$, St. Petersburg Math. J. 14 (2003), no. 5, 765-773.

[69] N. Kosovskiı̌, Gluing of Riemannian manifolds of curvature $\geq \kappa$, St. Petersburg Math. J. 14 (2003), no. 3, 467-478.

[70] N. Kosovskiı̌, Gluing with branching of Riemannian manifolds of curvature $\leq \kappa$, Algebra i Analiz 16 (2004), no. 4, 132-145 (Russian).

[71] A. Kuzminyh, Mappings preserving the distance 1, (Russian) Sibirsk. Mat. Zh. 20 (1979), no. 3, 597-602.

[72] U. Lang, Extendability of large scale lipschitz maps, Trans. AMS 351 (1999), 39753988.

[73] U. Lang, V. Schroeder, Kirszbraun's theorem and metric spaces of bounded curvature, Geom. and Funct. Anal., 7:3 (1997), 535-560.

[74] U. Lang, V. Schroeder, Jung's theorem for Aleksandrov spaces of curvature bounded above, Ann. Global Anal. Geom. 15 (1997), 263-275.

[75] U. Lang, B. Pavlović, V. Schroeder, Extensions of Lipschitz maps into Hadamard spaces, Geom. Funct. Anal. 10 (2000), no. 6, 1527-1553.

[76] B. Leeb, A characterization of irreducible symmetric spaces and Euclidean buildings of higher rank by their asymptotic geometry, Habilitationsschrift, Bonn 1997, Bonner math. Schriften 326 (2000).

[77] A. Lytchak, Geometry of sets of positive reach, Manuscripta Math. 115 (2004), 199-205.

[78] A. Lytchak, Differentiation in metric spaces, Algebra i Analiz 16 (2004), no. 6, 128-161; translation in St. Petersburg Math. J. 16 (2005), no. 6, 1017-1041.

[79] A. Lytchak, Almost convex subsets, Geom. Dedicata 115 (2005), 201-218.

[80] A. Lytchak, Rigidity of spherical buildings and joins, Geom. Funct. Anal. 15 (2005), $720-752$

[81] A. Lytchak, Strukur der Submetrien, PhD thesis, Bonn, 2001.

[82] A. Lytchak, K. Nagano, Geodesically complete spaces with an upper curvature bound, in preparation.

[83] A. Lytchak and V. Schroeder, Affine functions on CAT( $\kappa)$-spaces, Math. Z. 2006.

[84] Y. Mashiko, Convex functions on Alexandrov surfaces, Trans. A.M.S. 351 (1998), 3549-3567.

[85] E. McShane, Extension of range of functions, Bull. AMS 40 (1934), 837-842.

[86] C. Mese, The curvature of minimal surfaces in singular spaces, Comm. Anal. Geom. 9 (2001), 3-34.

[87] K. Nagano, Asymptotic rigidity of Hadamard 2-spaces, J. Math. Soc. Japan 52 (2000), no. 4, 699-723. 
[88] K. Nagano, A volume convergence theorem for Alexandrov spaces with curvature bounded above, Math. Z. 241 (2002), no. 1, 127-163.

[89] K. Nagano, A sphere theorem for 2-dimensional CAT(1)-spaces, Pacific J. Math. 206 (2002), no. 2, 401-423.

[90] I. Nikolaev, The tangent cone of an Aleksandrov space of curvature $\leq K$, Manuscripta Math. 86 (1995), 137-147.

[91] I. Nikolaev, A metric characterization of Riemannian spaces, Siberian Adv. Math. 9 (1999), no. 4, 1-58.

[92] I. Nikolvaev, On the Sasaki distance between directions in a metric space and solution of a problem by A.D. Aleksandrov on synthetic description of Riemannian manifolds, In book: Communications of International School-Conference on Analysis and Geometry. Novosibirsk 2004, (2004), 23-27.

[93] I. Nikolaev, Space of directions at a point of a space of curvature not greater than K, Siberian Math. J. 19 (1978), no. 6, 944-949.

[94] Yu. Otsu, Differential geometric aspects of Alexandrov spaces, Comparison Geometry (K. Grove and P. Petersen, eds.), M.S.R.I. Publ. 30, Cambridge Univ. Press, 1997, 135-148.

[95] Yu. Otsu, T. Shioya, The Riemannian structure of Alexandrov spaces, J. Diff, Geom. 39 (1994), no. 3, 629-658.

[96] A. Petrunin, Applications of quasigeodesics and gradient curves, In Comparison Geometry, pages 203-219. Berkeley, CA, 1993-94.

[97] A. Petrunin, Metric minimizing surfaces, Electron. Res. Announc. Amer. Math. Soc. 5 (1999), 47-54.

[98] Yu. Reshetnyak, On the theory of spaces of curvature not greater than $K$, Mat. Sbornik, 52 (1960), 789-798.

[99] Yu. Reshetnyak, Two-dimensional manifolds of bounded curvature, Geometry. IV. Nonregular Riemannian geometry, Encyclopaedia Math. Sci., vol. 70, SpringerVerlag, Berlin, 1993, 3-163.

[100] Yu. Reshetnyak, Nonexpanding maps in a space of curvature no greater than K, Sib. Mat. Zh. 9 (1968), 918-928 (Russian). English translation: Inextensibly mappings in a space of curvature no greater than K, Siberian Math. J. 9 (1968), 683-689.

[101] S. Sasaki, On the differential geometry of tangent bundles of Riemannian manifolds, Tôhoku Math. J. (2) 10 (1958), 338-354.

[102] S. Sasaki, On the differential geometry of tangent bundles of Riemannian manifolds. II, Tôhoku Math. J. (2) 14 (1962), 146-155.

[103] J. Simons, On transitivity of holonomy systems, Ann. of Math. 76 (1962), 213-234.

[104] P. Thurston, CAT(0) 4-manifolds possessing a single tame point are Euclidean, J. Geom. Anal. 6 (1996), 475-494.

Steklov Institute of Mathematics, Fontanka 27, 191011,

St. Petersburg, Russia

E-mail address: sbuyalo@pdmi.ras.ru

Institut für Mathematik, Universität Zürich, Winterthurer Strasse 190, CH-8057 ZÜRICH, SwitZERLAND

E-mail address: vschroed@math.unizh.ch 
